\title{
Cascade Sliding Control for Trajectory Tracking of a Nonholonomic Mobile Robot with Adaptive Neural Compensator
}

\author{
Flavio Capraro, ${ }^{1}$ Francisco Guido Rossomando, ${ }^{1}$ Carlos Soria, ${ }^{1}$ and Gustavo Scaglia ${ }^{2}$ \\ ${ }^{1}$ Instituto de Automática-CONICET, Facultad de Ingeniería, Universidad Nacional de San Juan, \\ Av. San Martín (Oeste) 1109, San Juan, Argentina \\ ${ }^{2}$ Instituto de Ingeniería Química-CONICET, Facultad de Ingeniería, Universidad Nacional de San Juan, \\ Av. San Martín (Oeste) 1109, San Juan, Argentina \\ Correspondence should be addressed to Francisco Guido Rossomando; frosoma@inaut.unsj.edu.ar
}

Received 31 March 2017; Revised 12 July 2017; Accepted 24 July 2017; Published 19 September 2017

Academic Editor: Francisco Valero

Copyright (C) 2017 Flavio Capraro et al. This is an open access article distributed under the Creative Commons Attribution License, which permits unrestricted use, distribution, and reproduction in any medium, provided the original work is properly cited.

A design of sliding mode controllers (SMC) with adaptive capacity is presented. This control technique is formed by two cascaded SMC controllers, one of them having an adaptive neural compensator (ANC); both are put on a WMR (wheeled mobile robot). The mobile robot is divided into a kinematics and a dynamics structure; the first SMC controller acts only on the kinematic structure and the SMC with neural adaptive compensator on the other one. The dynamic SMC was designed applying an inverse dynamic controller and using the model dynamics of the WMR. The adaptive neural compensation (ANC) was used in order to reduce the control error caused by the dynamics variations but it conveys a residual approximation error, so a sliding part was designed to cancel such error. This technique allows achieving the control objective despite parameter variations and external disturbances that take place in the dynamics; on the other hand, the ANC can adjust its neural parameters to reduce the dynamics variations of the WMR and thus improve the trajectory tracking control. Problems of convergence and stability are treated and design rules based on Lyapunov's theorem are given.

\section{Introduction}

1.1. Previous Works. The problem of trajectory tracking is a classic control one; its importance lies in the actual applications that these algorithms have. Although some of these problems have been solved before, there is always the possibility of somehow improving the algorithms previously designed.

The biggest advantage in using SMC technique is the low sensitivity in front of changes in the process dynamics and external disturbances, which eliminates the necessity of an accurate model. Recent works about disturbance rejections and SMC applied to robots control are dealt with in papers such as [1], where a spherical robot using Omni wheels uses a cascade sliding mode control (CSMC) to control its position.

The structure of the CSMC is formed by a cascade combination of states of the dynamic model and a set of sliding surfaces. This work shows that the problem of constant speed can be avoided in order to achieve the position control without adaptive control techniques. An application of SMC in WMR is presented in [2]; the trajectory tracking problem is investigated for a nonholonomic wheeled mobile robot (NWMR) with parameter uncertainties and external disturbances. In this strategy, combining the kinematic model with the dynamic model, the actuator voltage is employed as the control input, and the uncertainties are approximated by a fuzzy logic system. The control system is stable and the tracking position errors converge asymptotically to zeros. Simulation results demonstrate the effectiveness of the proposed strategy.

In similar form, Ashrafiuon et al. [3] presented a nonlinear sliding mode control law that is employed to stabilize the error dynamics. It is shown that the control law is uniformly asymptotically stable if unknown disturbances and modeling 
uncertainties are bounded. The framework is applied to differential drive mobile robots, air vehicles operating in the vertical plane, and marine vehicles. Simulations are presented for models of in-house mobile robots and surface vessels subject to unknown disturbances.

Another design for SMC controllers is proposed by [4], who uses a controller based on linear algebra for mobile robots. The results presented show a good performance, while the oscillations in trajectory tracking are reduced.

In the work presented by [5], the tracking control of a two-wheeled mobile robot is considered to underpin the developed theoretical results based on SMC. Model-based tracking control of a wheeled mobile robot (WMR) is first transferred to a stabilization problem for the corresponding tracking error system, and then the developed theoretical results are applied to show that the tracking error system is globally asymptotically stable even in the presence of matched and mismatched uncertainties. These works [3-5] do not present adaptive capacity combined with SMC in trajectory tracking control.

In another work [6], a practical control law for wheeled mobile robots is proposed in order to improve the transient performance and decrease the tracking errors. The nominal system is governed using a controller derived under the back-stepping framework. Such a design can effectively reach the system's tracking objective and enhance robustness via properly configured parameters.

Another case of cascade-control algorithm based on a sliding mode is proposed by [7]. It is implemented for trajectory tracking control of a hydraulically driven 6-DOF (six degrees of freedom) robot manipulator. The proposed controller, which consists of two control loops, is applied to separate the hydraulic dynamics from the mechanical part so that the designed controller takes into account not only the mechanical dynamics but also the hydraulic dynamics of the manipulator. Experimental results demonstrate the satisfactory position tracking behavior of the parallel manipulator using a cascade controller based on a sliding mode.

In the meantime, a two-time-scale filter is applied to the system function to estimate the disturbances, essentially improving the system's precision. Experimental results demonstrated that the proposed control is practical for WMRs in tracking control. Instead in [8] the authors present a SMC for the whole structure of the mobile robot and the results show a good performance as well as asymptotic convergence demonstrated using Lyapunov's theory. But the control law is not trivial to implement for another kind of mobile robot. Similar research is shown in [9], where an adaptive neural network DSC (dynamic surface control) formulated on disturbance observer for the WMR (wheeled mobile robot) with parameters variations and external disturbances has been implemented. The observer is employed to estimate the unknown disturbances, and the neural approximation is used to identify the nonlinear model and all possible uncertainties of the system. Then, the Lyapunov theory is used to demonstrate the convergence of the proposed control system. Simulation and experiments results are included to demonstrate the adaptive capability and robustness of the proposed control approach.
In [10], an adaptive exponential SMC is proposed as a possible solution to minimize the chattering effect, external disturbances, and parameters variations in trajectory tracking of a wheeled mobile robot. The results obtained via experimentation show the effectiveness of the adaptive exponential SMC against disturbances and parameters variations.

Another important consideration in SMC is how to reduce the chattering effect; in [11] passivity-based adaptive and nonadaptive chattering-free sliding mode controllers are proposed assuming that the norm upper bound of the derivative of the sliding surface is available.

In [12], a method based on an integral SMC applied in the trajectory tracking of WMR is presented. The control technique is designed to solve the reaching phase problem with the elimination of matched disturbances and minimize the unmatched one. The proposed controller has two parts: first one is a high-level stabilization controller to compensate the known system and second one is a supervisory controller to improve the trajectory tracking in the presence of disturbances. The simulation results are based on the kinematics model of the nonholonomic mobile robot.

1.2. Contribution. In this work, an adaptive neural compensation (ANC) is considered. The ANC can be used by static controllers, obtaining as a result an adaptive control over the whole robotic system to be controlled. In this case, the ANC is applied over an inverse dynamic controller, and the complete control system shows a similar behavior of an adaptive controller system.

The paper [13] describes a method for the design of the discrete time dynamic compensation for a WMR with parameters variations, and the application of this method to trajectory tracking control. This method uses a single NN-RBF for dynamic compensation and is designed using Lyapunov's criterion. The stability analysis demonstrates a Uniformly Ultimately Bounded (UUB) control method.

Another work [14] shows a control technique based on a SMC and adaptive neural control applied over a WMR, but the sliding control is acting over the whole dynamic part of WMR.

This paper deals with the adaptive trajectory tracking control using SMC, and its design is based on a nominal robot dynamics. The control system has two parts. The first one uses the robot kinematics and SMC. The second one uses the WMR dynamics to design a nominal inverse control with ANC.

The main assumption of this work is that the most important parametric variations and uncertainties of the WMR appear in the dynamic part. The control technique uses a neural net based on RBF (radial basis functions) in combination with SMC and inverse dynamics. The control part based on inverse dynamics is designed on the nominal model of the WMR.

The adaptation laws of the ANC are obtained using Lyapunov's stability criterion, obtaining an asymptotic stability of the proposed control system. 


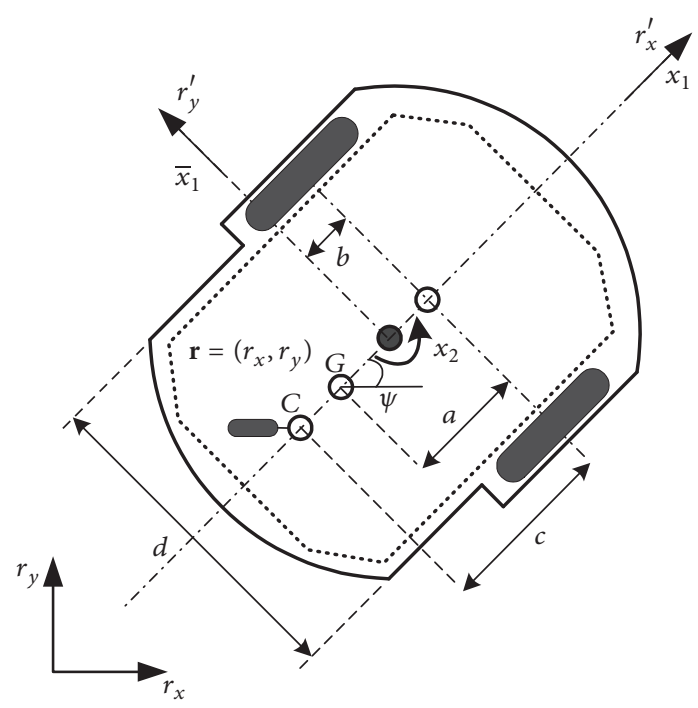

FIGURE 1: Mobile robot parameters distribution.

The combination of cascade SMC and ANC technique presents the following advantages:

(1) The focus of this technique is the application of two sliding controllers in cascade to reduce problems on the perfect velocity matching of the WMR caused by parameters or dynamics variations. And it can be applied in WMR systems or nonlinear systems when their parameters vary or are unknown.

(2) The ANC does not need to learn the whole robot structure; it adjusts the dynamic variations to approximate the nominal to the real dynamics of the WMR.

(3) The ANC is easy to implement and it can be applied in different controllers without adaptive capacity, obtaining an adaptive control technique for the proposed control problem.

1.3. Organization. The paper is presented as follows. In Section 2, a description of the WMR system is given and the robot model (kinematic and dynamic parts) is proposed. Explicit expressions of the cascade SMC and the ANC compensation are derived in Sections 3 and 4, respectively. These expressions are used to obtain the neural adjustment law and prove the convergence of the proposed control system (Section 5). Several experimental results are presented in Section 6 for the evaluation of the proposed control technique and demonstrate the effectiveness of the adaptive capability. Finally, Section 7 concludes the paper.

\section{Robot System Description}

Figure 1 shows a typical structure of a nonholonomic mobile robot. It consists of a robot body (CPU, sensors, and motors) with two driving wheels mounted on the same axis and two motors for each one and a free rear wheel (or castor wheel). Both motors are the actuators of motion and orientation and provide the torque to the wheels.

For WMR control, the variables of interest in inertial Cartesian frame (XY plane) are $r_{x}, r_{y}, \psi, x_{1}$, and $x_{2}$, where $r_{x}, r_{y}$ are the coordinates of the point of interest $\mathbf{r}$, the angle $\psi$ indicates the WMR direction, and the pair $x_{1}, x_{2}$ is the linear and angular velocities, respectively, of the WMR.

The WMR parameters are described as follows:

$G$ denotes the mass center of the WMR.

$c$ is the distance between the free wheel (castor wheel) referred from the axis of the traction wheels.

$a$ is the distance between the $G$ point and the axis of the traction wheels.

The robot's model is divided taking into account the kinematics and the dynamics models, as shown in Figure 2. The kinematic and dynamic models were formulated for [15] and can be expressed as follows.

Kinematics Model

$$
\begin{aligned}
\left(\begin{array}{c}
\dot{r}_{x}(t) \\
\dot{r}_{y}(t) \\
\dot{\psi}(t)
\end{array}\right)= & \left(\begin{array}{cc}
\cos \psi(t) & -a \sin \psi(t) \\
\sin \psi(t) & a \cos \psi(t) \\
0 & 1
\end{array}\right)\left(\begin{array}{l}
x_{1}(t) \\
x_{2}(t)
\end{array}\right) \\
& +\left(\begin{array}{c}
\delta_{r x}(t) \\
\delta_{r y}(t) \\
0
\end{array}\right) .
\end{aligned}
$$

Dynamic Model

$$
\begin{aligned}
\left(\begin{array}{c}
\dot{x}_{1}(t) \\
\dot{x}_{2}(t)
\end{array}\right)= & \left(\begin{array}{c}
\frac{\kappa_{3}}{\kappa_{1}} x_{2}^{2}(t)-\frac{\kappa_{4}}{\kappa_{1}} x_{1}(t) \\
-\frac{\kappa_{5}}{\kappa_{2}} x_{1}(t) x_{2}(t)-\frac{\kappa_{6}}{\kappa_{2}} x_{2}(t)
\end{array}\right) \\
& +\left(\begin{array}{cc}
\frac{1}{\kappa_{1}} & 0 \\
0 & \frac{1}{\kappa_{2}}
\end{array}\right)\left(\begin{array}{l}
u_{1}(t) \\
u_{2}(t)
\end{array}\right)+\left(\begin{array}{l}
\delta_{x 1}(t) \\
\delta_{x 2}(t)
\end{array}\right) .
\end{aligned}
$$

The identified parameters vector $\boldsymbol{\kappa}=\left[\kappa_{1}, \kappa_{2}, \kappa_{3}, \kappa_{4}, \kappa_{5}, \kappa_{6}\right]^{T}$ is described in the Appendix and the model uncertainties of the WMR are the pair $\left(\delta_{r x}, \delta_{r y}\right)$ and the pair $\left(\delta_{x 1}, \delta_{x 2}\right)$, where the pair $\left(\delta_{r x}, \delta_{r y}\right)$ is dependent on WMR friction and slip velocities and some of these uncertainties influences on mobile robots are described in [16]. On the other hand, the pair $\left(\delta_{x 1}, \delta_{x 2}\right)$ is dependent on robot's parameters as load weight, mass, inertia, wheel diameter, tires properties (static and dynamic friction), electric motor, its servos, and others.

\section{Sliding Mode Kinematics Controller}

To obtain the kinematics controller, the robot's kinematics model is necessary to know. The robot's kinematics model (2) can be rewritten as follows:

$$
\begin{aligned}
\dot{\mathbf{r}}(t) & =\mathbf{L}(t) \mathbf{x}(t)+\boldsymbol{\delta}(t) \\
& =\left(\begin{array}{cc}
\cos \psi(t) & -a \sin \psi(t) \\
\sin \psi(t) & a \cos \psi(t)
\end{array}\right) \mathbf{x}(t)+\left(\begin{array}{c}
\delta_{r x}(t) \\
\delta_{r y}(t)
\end{array}\right) .
\end{aligned}
$$




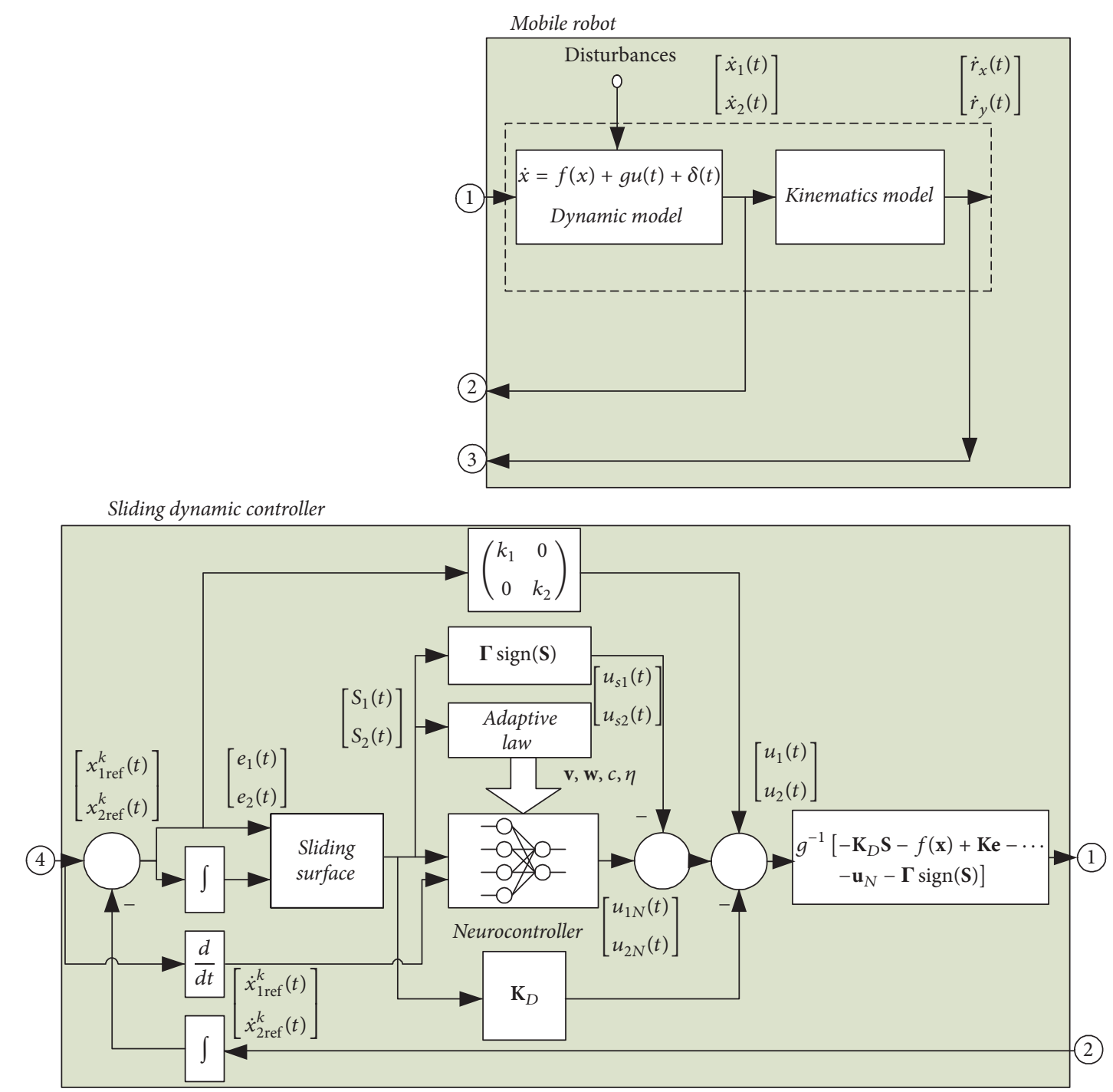

Sliding kinematic controller

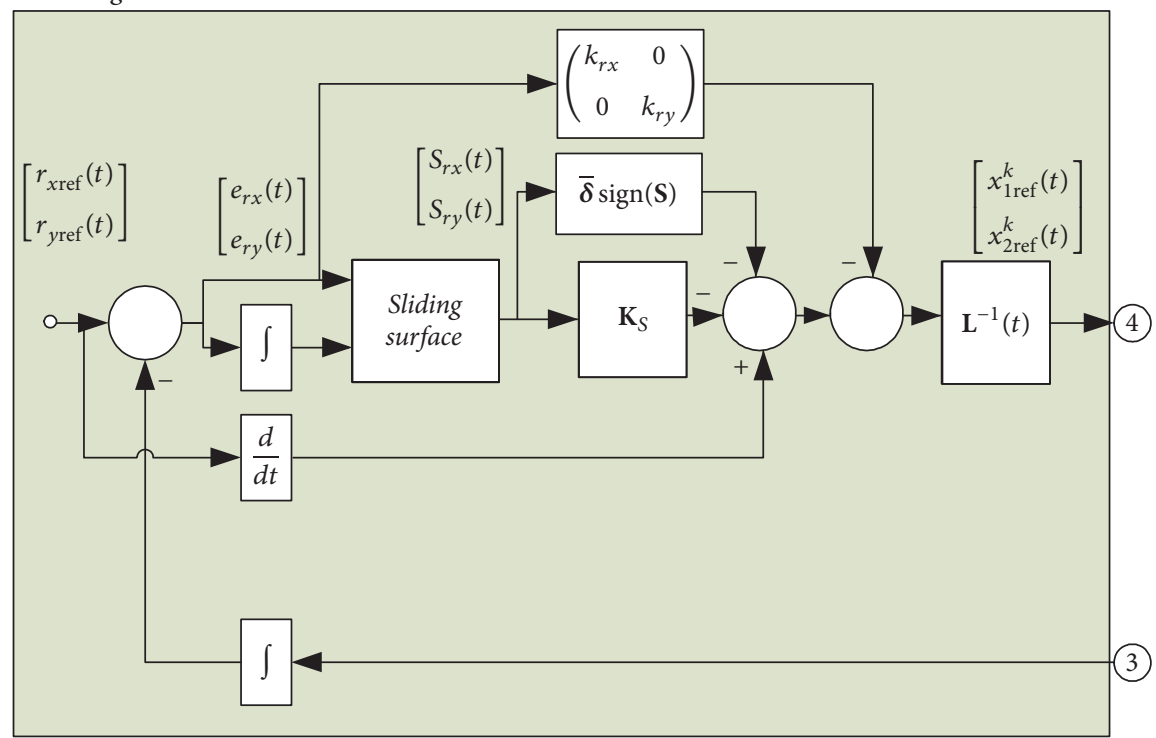

FIgURE 2: Structure control of CSMC with ANC. 
For (3) to be controllable, it requires that $\mathbf{L}(t)$ must be nonsingular.

Assumption 1. $\boldsymbol{\delta}_{\mathbf{r}}(t)$ is a nonmodeled kinematics vector which is bounded, where $\bar{\delta}$ is the upper bound.

$$
\bar{\delta}=\sup _{t \in \Re^{+}}|\boldsymbol{\delta}(t)|,
$$

where

$$
\begin{aligned}
\mathbf{r}(t) & =\left(\begin{array}{ll}
r_{x}(t) & r_{y}(t)
\end{array}\right)^{T} ; \\
\mathbf{x}(t) & =\left(\begin{array}{ll}
x_{1}(t) & x_{2}(t)
\end{array}\right)^{T} ; \\
\boldsymbol{\delta}(t) & =\left(\begin{array}{lll}
\delta_{r x}(t) & \delta_{r y}(t)
\end{array}\right)^{T} .
\end{aligned}
$$

They are the position output vector, the velocities input vector, and the vector of nonmodeled kinematics. The trajectory tracking error can be expressed as

$$
\begin{aligned}
\mathbf{e}_{r}(t) & =\mathbf{r}(t)-\mathbf{r}_{\text {ref }}(t) \\
& =\left(r_{x}(t)-r_{x \mathrm{ref}}(t), r_{y}(t)-r_{y \mathrm{ref}}(t)\right)^{T} .
\end{aligned}
$$

The main objective of the control law, which can be obtained by the SMC, is the convergence of any state trajectory of nonlinear system to specified surface (defined by designer) into space state and maintain it in the subsequent time [1719].

A sliding surface for a MIMO system can be obtained by position error (6) and defining $\mathbf{S}_{\mathbf{r}}=\left(S_{r x}, S_{r y}\right)^{T}$ as

$$
\begin{aligned}
S_{r i}(\mathbf{r}) & =\left(\frac{d}{d t}+k_{r i}\right) \int_{0}^{t} e_{r i}(\tau) d \tau \\
& =e_{r i}(t)+k_{r i} \int_{0}^{t} e_{r i}(\tau) d \tau \text { for } i=x, y .
\end{aligned}
$$

The derivative of (7) is

$$
\begin{aligned}
\dot{S}_{r i}(\mathbf{r}) & =\frac{d}{d t}\left(e_{r i}(t)+k_{r i} \int_{0}^{t} e_{r i}(\tau) d \tau\right) \\
& =\dot{e}_{r i}(t)+k_{r i} e_{r i}(t) .
\end{aligned}
$$

$k_{r i}$ is a strictly positive constant and $S_{r i}$ denotes a row of $\mathbf{S}_{\mathbf{r}}$ vector. In the design of the kinematics SMC, first, an ideal equivalent control law $\mathbf{x}_{\text {ref }}^{k}(t)$ must be determined. The ideal control law is obtained from (8) equaling zero as

$$
\dot{\mathbf{S}}_{r}=\left(\begin{array}{l}
\dot{S}_{r x} \\
\dot{S}_{r y}
\end{array}\right)=\left(\begin{array}{l}
0 \\
0
\end{array}\right) \text {. }
$$

Replacing (3) with (8), equaling zero, the following is obtained:

$$
\begin{aligned}
\dot{\mathbf{e}}_{r}( & (t)+\operatorname{diag}\left(k_{r x}, k_{r y}\right) \mathbf{e}_{r}(t) \\
= & \left(\mathbf{L x}(t)+\boldsymbol{\delta}(t)-\dot{\mathbf{r}}_{\mathrm{ref}}(t)\right)+\operatorname{diag}\left(k_{r x}, k_{r y}\right) \mathbf{e}_{r}(t) \\
= & 0 .
\end{aligned}
$$

Similar case was studied in [17], defining the control law $\mathbf{x}_{\text {ref }}^{k}(t)$ that guarantees the sliding condition (9), which is composed of an ideal control $\mathbf{x}_{\text {ref }}^{k}(t)=\mathbf{L}^{-1}\left[-\operatorname{diag}\left(k_{r x}, k_{r y}\right) \mathbf{e}_{r}(t)-\right.$ $\left.\boldsymbol{\delta}_{r}(t)+\dot{\mathbf{r}}_{\text {ref }}(t)\right]$, but the term $\boldsymbol{\delta}_{r}$ is unknown; taking into account Assumption 1, $\boldsymbol{\delta}_{r}$ is bounded. For control purposes, it can be approximated by $-\overline{\boldsymbol{\delta}} \operatorname{sign}(\mathbf{S})$. Thus, the control law is defined by

$$
\begin{aligned}
& \mathbf{x}_{\mathrm{ref}}^{k}(t)=\mathbf{L}^{-1}(t)\left[-\mathbf{K}_{S} \mathbf{S}_{r}-\operatorname{diag}\left(k_{r x}, k_{r y}\right) \mathbf{e}_{r}(t)\right. \\
& \left.-\overline{\boldsymbol{\delta}} \operatorname{sign}(\mathbf{S})+\dot{\mathbf{r}}_{\mathrm{ref}}(t)\right] .
\end{aligned}
$$

Then the values of $\mathbf{L}^{-1}, \operatorname{sign}\left(S_{i}\right)$, and $\mathbf{K}_{S}$ are

$$
\begin{aligned}
\mathbf{L}^{-1} & =\left(\begin{array}{cc}
\cos \psi(t) & \sin \psi(t) \\
-\frac{1}{a} \sin \psi(t) & \frac{1}{a} \cos \psi(t)
\end{array}\right) \\
\operatorname{sgn}\left(S_{i}\right) & = \begin{cases}1 & \text { for } S_{i}>0 \\
0 & \text { for } S_{i}=0 \\
-1 & \text { for } S_{i}<0,\end{cases} \\
\mathbf{K}_{S} & =\left(\begin{array}{cc}
K_{S x} & 0 \\
0 & K_{S y}
\end{array}\right) .
\end{aligned}
$$

The term $\left(-\mathbf{K}_{S} \mathbf{S}\right)$ was included to guarantee the convergence of (11); now we define Lyapunov's function candidate like

$$
V^{k}=\frac{\mathbf{S}_{r}^{T} \mathbf{S}_{r}}{2},
$$

where the superscript $k$ denotes the analysis performed with respect to the kinematic controller; now differentiating (13) with respect to time and replacing (3) lead to

$$
\begin{aligned}
\dot{V}^{k} & =\mathbf{S}_{r}^{T} \dot{\mathbf{S}}_{r}=\mathbf{S}_{r}^{T}\left(\dot{\mathbf{e}}_{r}(t)+\operatorname{diag}\left(k_{r x}, k_{r y}\right) \mathbf{e}_{r}(t)\right) \\
& =\mathbf{S}^{T}\left(\left(\dot{\mathbf{r}}(t)-\dot{\mathbf{r}}_{\text {ref }}(t)\right)+\operatorname{diag}\left(k_{r x}, k_{r y}\right) \mathbf{e}_{r}(t)\right)=\cdots \\
& =\mathbf{S}_{r}^{T}\left(\left(\mathbf{L x}(t)+\boldsymbol{\delta}(t)-\dot{\mathbf{r}}_{\text {ref }}(t)\right)\right. \\
& \left.+\operatorname{diag}\left(k_{r x}, k_{r y}\right) \mathbf{e}_{r}(t)\right) .
\end{aligned}
$$

Replacing (11) with (14),

$$
\begin{aligned}
\dot{V}^{k} & =\mathbf{S}_{r}^{T}\left(\left(-\mathbf{K}_{S} \mathbf{S}_{r}+\boldsymbol{\delta}(t)-\bar{\delta} \operatorname{sign}(\mathbf{S})\right)\right. \\
& \left.+\operatorname{diag}\left(k_{r x}, k_{r y}\right) \mathbf{e}_{r}(t)\right) \leq-\left\|\mathbf{K}_{S}\right\|\left\|\mathbf{S}_{r}\right\|^{2}+\|\boldsymbol{\delta}\|\left\|\mathbf{S}_{r}\right\| \\
& -\bar{\delta}\left\|\mathbf{S}_{r}\right\| .
\end{aligned}
$$

Then, the following is obtained:

$$
\dot{V}^{k} \leq-\left\|\mathbf{K}_{S}\right\|\left\|\mathbf{S}_{r}\right\|^{2}+\Delta_{r}\left\|\mathbf{S}_{r}\right\|<0 \quad \text { for } S_{i} \neq 0 .
$$

From Assumption $1\left(\left\|\boldsymbol{\delta}_{r}\right\|-\bar{\delta}\right)=\Delta_{r} \leq 0$ and $\dot{V}^{k}(t)$ is negative definite; now integrating both sides of (16) results in

$$
\begin{aligned}
& \int_{0}^{t s}\left(\left\|\mathbf{K}_{S}\right\|\left\|\mathbf{S}_{r}\right\|^{2}+\Delta_{r}\left\|\mathbf{S}_{r}\right\|\right) d t=-\int_{0}^{t s} \dot{V}^{k}(t) d t \\
& =V^{k}(0)-V^{k}(t s) .
\end{aligned}
$$


Because $V^{k}(0)$ is bounded and $V^{k}(t s)$ is also bounded and nonincreasing, it can be obtained that

$$
\lim _{t s \rightarrow \infty}\left[-\int_{0}^{t s} \dot{V}^{k}(t) d t\right]=V^{k}(0)-V^{k}(t s)<\infty .
$$

By Barbalat's lemma [17], it can be shown that limit $\lim _{t \rightarrow \infty}\left[\dot{V}^{k}(t)\right]$. That is, $\mathbf{S}_{r}(t)$ tends to zero as $t$ tends to infinity, being a stable control system. Therefore, the tracking error of the SMC based on WMR kinematics tends to zero as $\mathbf{S}_{r}(t)$ tends to zero.

\section{Sliding Mode Controller with Adaptive Neural Compensation}

4.1. Problem Formulation. At this point, new solutions based on SMC and ANC for WMR considering parameter variations in its dynamics are proposed. The aim of this work is to reduce the trajectory tracking error produced by the uncertainties in the WMR model and parameter variations in the dynamics, but maintaining an adequate mechanical performance and improving the performance of the WMR during trajectory tracking.

The proposed strategy in this matter combines inverse dynamics control and adaptive neural compensation (ANC) with SMC, where the inverse dynamics controller is built with the known part of the robot's dynamics, the adaptive neural part compensates dynamic variations of the robot, and SMC eliminates the error introduced by the neural network.

This point aims to design a combination of SMC and ANC which ensure the global convergence of controlled variables for the control system for a given reference trajectory $\mathbf{x}_{\mathrm{ref}}^{k}(t)$.

Now, the system model (2) is expressed in compact form as

$$
\dot{\mathbf{x}}=f(\mathbf{x})+\Delta f(\mathbf{x})+(g+\Delta g(\mathbf{x})) \mathbf{u},
$$

where

$$
\begin{aligned}
& \dot{\mathbf{x}}(t)=\left(\begin{array}{c}
\dot{x}_{1} \\
\dot{x}_{2}
\end{array}\right) ; \\
& f(\mathbf{x})=\left(\begin{array}{l}
f_{1}(\mathbf{x}) \\
f_{2}(\mathbf{x})
\end{array}\right)=\left(\begin{array}{c}
\frac{\kappa_{3}}{\kappa_{1}} x_{2}^{2}(t)-\frac{\kappa_{4}}{\kappa_{1}} x_{1}(t) \\
-\frac{\kappa_{5}}{\kappa_{2}} x_{1}(t) x_{2}(t)-\frac{\kappa_{6}}{\kappa_{2}} x_{2}(t)
\end{array}\right) ; \\
& g \mathbf{u}(t)=\left(\begin{array}{cc}
g_{1} & 0 \\
0 & g_{2}
\end{array}\right)\left(\begin{array}{l}
u_{1} \\
u_{2}
\end{array}\right)=\left(\begin{array}{cc}
\frac{1}{\kappa_{1}} & 0 \\
0 & \frac{1}{\kappa_{2}}
\end{array}\right)\left(\begin{array}{l}
u_{1} \\
u_{2}
\end{array}\right) \\
& =\left(\begin{array}{l}
\frac{u_{1}}{\kappa_{1}} \\
\frac{u_{2}}{\kappa_{2}}
\end{array}\right) \\
& \Delta f(\mathbf{x})+\Delta g(\mathbf{x}) \mathbf{u}(t)=\left(\begin{array}{l}
\delta_{x 1}(\mathbf{x}) \\
\delta_{x 2}(\mathbf{x})
\end{array}\right),
\end{aligned}
$$

where $f_{i}(\cdot), g_{i}(\cdot)$ denote row vectors of $f(\cdot)$ and $g(\cdot)$, respectively, $\mathbf{x}=\left[x_{1}, x_{2}\right]^{T} \in \mathfrak{R}^{n}$ is the state vector, and $\mathbf{u}=$ $\left[u_{1}, u_{2}\right]^{T} \in \mathfrak{R}^{n}$ with $n=2$ is the control action input vector.

Assumption 2. Dynamic variations in (19) produce another pair of additive functions represented by $\Delta f(\mathbf{x})$ and $\Delta g(\mathbf{x})$, which represent nonlinear unknown functions and are considered as uncertainties.

The WMR dynamics from (1) without its variation is given by

$$
\dot{\mathbf{x}}=f(\mathbf{x})+g \mathbf{u}
$$

Assumption 3. The matrix $\mathbf{g}$ is nonsingular; that is, $\mathbf{g}^{-1}$ exists and has bounded norm; this is equivalent to assuming

$$
\begin{aligned}
\lambda_{l}(\mathbf{g}) & >\lambda_{\min }>0, \\
\|\mathbf{g}\|_{2} & =\lambda_{p}(\mathbf{g}) \leq \lambda_{\max }<\infty,
\end{aligned}
$$

where $\lambda_{l}(\mathbf{g})$ and $\lambda_{p}(\mathbf{g})$ are the lower and upper eigenvalues of g, respectively.

4.2. Implementation of SMC with Adaptive Neural Compensation (ANC). The objective of this issue is to design a control law that combines SMC with adaptive neural compensation. And this control law must ensure the convergence of all controlled variables. The SMC with ANC which is designed based on the NN-RBF structure can deal with this kind of control problem [20].

For a good trajectory tracking, the output $\mathbf{x}(t)$ must follow the desired trajectory $\mathbf{x}_{\text {ref }}^{k}(t)$; therefore, the tracking error should converge to zero.

Now, we define the tracking error as

$$
\mathbf{e}=\mathbf{x}-\mathbf{x}_{\mathrm{ref}}^{k}=\left(x_{1}-x_{\mathrm{ref} 1}^{k}, x_{2}-x_{\mathrm{ref} 2}^{k}\right)^{T}=\left(e_{1}, e_{2}\right)^{T} .
$$

A sliding surface $\mathbf{S}(\mathbf{x})=\left(S_{1}, S_{2}\right)^{T}$ for the WMR system can be written in terms of the control error,

$$
\begin{aligned}
S_{i}(\mathbf{x}) & =\left(\frac{d}{d t}+k_{i}\right) \int_{0}^{t} e_{i}(\tau) d \tau \\
& =e_{i}(t)+k_{i} \int_{0}^{t} e_{i}(\tau) d \tau \quad \text { for } i=1,2 .
\end{aligned}
$$

The derivate of (24) is

$$
\begin{aligned}
\dot{S}_{i}(\mathbf{x}) & =\frac{d}{d t}\left(e_{i}(t)+k_{i} \int_{0}^{t} e_{i}(\tau) d \tau\right) \\
& =\left(\dot{x}_{i}-\dot{x}_{\text {ref } i}\right)+k_{i} e_{i} .
\end{aligned}
$$

To ensure that the state variables remain on the sliding surface, it is necessary for $\dot{S}_{i}(\mathbf{x})$ to equal zero,

$$
\dot{S}_{i}(\mathbf{x})=\left(f_{i}(\mathbf{x})+g_{i} u_{i}-\dot{x}_{\text {refi }}\right)+k_{i} e_{i}=0 .
$$

Based on (26), the equivalent control law $\mathbf{u}(t)$ can be obtained, and in the same way of the previous section, we 
included the term $K_{D i} S_{i}$ to guarantee the convergence, and the obtained control law is expressed as

$$
u_{i}(t)=\left(g_{i}\right)^{-1}\left(-K_{D i} S_{i}+\dot{x}_{\text {refi }}-f_{i}(\mathbf{x})-k_{i} e_{i}\right) .
$$

Now, it is necessary to define a control law for the nonlinear system (21), define a new control law $\mathbf{u}$ that guarantees robust performance and the convergence, and also ensure the sliding condition [20]. This new control law is implemented by (27) and a discontinuous term $-\Gamma \operatorname{sign}(\mathbf{S})$. It is defined by

$$
\begin{aligned}
& u_{i}(t) \\
& \quad=\kappa_{i}\left[-K_{D i} S_{1}+\dot{x}_{\text {refi }}-f_{i}(\mathbf{x})-k_{i} e_{i}-\Gamma_{i} \operatorname{sign}\left(S_{i}\right)\right] .
\end{aligned}
$$

From (20), $g_{i}^{-1}=\kappa_{i}$ because $g$ is a diagonal matrix and the term $\Gamma_{i} \operatorname{sign}\left(S_{i}\right)$ is constituted by a strictly positive constant $\Gamma_{i}$ and a discontinuity $\left(\operatorname{sign}\left(S_{i}\right)\right)$. The function $\operatorname{sign}(\cdot)$ is defined by

$$
\operatorname{sign}\left(S_{i}\right)= \begin{cases}1 & \text { for } S_{i}>0 \\ 0 & \text { for } S_{i}=0 \\ -1 & \text { for } S_{i}<0\end{cases}
$$

To prove the convergence of (28), a Lyapunov's function candidate is defined as

$$
V=\frac{1}{2}\left(\mathbf{S}^{T} \mathbf{S}\right)=\sum_{i=1}^{2} \frac{1}{2}\left(S_{i}^{2}\right) .
$$

Differentiating (30) with respect to time is expressed by

$$
\begin{aligned}
\dot{V} & =\sum_{i=1}^{2} S_{i} \dot{S}_{i}=\sum_{i=1}^{2} S_{i}\left(\frac{d}{d t} e_{i}(t)+k_{i} e_{i}(t)\right) \\
& =\sum_{i=1}^{2} S_{i}\left(\left(\dot{x}_{i}-\dot{x}_{\text {refi }}\right)+k_{i} e_{i}(t)\right)=\cdots \\
& =\sum_{i=1}^{2} S_{i}\left(f_{i}(\mathbf{x})+g_{i} u_{i}-\dot{x}_{\text {ref } i}+k_{i} e_{i}(t)\right),
\end{aligned}
$$

and replacing (28) with (31),

$$
\begin{aligned}
\dot{V} & =\sum_{i=1}^{2} S_{i} \dot{S}_{i}=\sum_{i=1}^{2} S_{i}\left(-K_{D i} S_{i}-\Gamma_{i} \operatorname{sgn}\left(S_{i}\right)\right) \\
& \leq-\sum_{i=1}^{2}\left(K_{D i} S_{i}^{2}+\Gamma_{i}\left|S_{i}\right|\right)<0 .
\end{aligned}
$$

Equation (32) proves the convergence to zero of the designed control law (28). The next step is considering the model indicated in (19) with dynamic variations; the nonmodeled structure can be expressed as

$$
\dot{x}_{i}=\left(f_{i}(\mathbf{x})+\Delta f_{i}(\mathbf{x})\right)+\left(\left(g_{i}+\Delta g_{i}(\mathbf{x})\right) u_{i}\right) \text {. }
$$

Substituting the dynamic part of the WMR (33) into (26) and applying the proposed control action (28), the following is obtained:

$$
\dot{S}_{i}(\mathbf{x})=-K_{D i} S_{i}+\Delta f_{i}(\mathbf{x})+\Delta g_{i}(\mathbf{x}) u_{i}-\Gamma_{i} \operatorname{sign}\left(S_{i}\right) .
$$

In (34), the terms corresponding to $\Delta f_{i}(x)$ and $\Delta g_{i}(x)$ disturbances are undesired; to deal with this problem, the $u_{i N}(t)$ term with adaptive capacity is added, which would compensate $\Delta f_{i}(x)$ and $\Delta g_{i}(x)$ variations in the dynamics of the system. And the term $\Gamma_{i} \operatorname{sign}\left(S_{i}\right)$ would eliminate the undesired errors (approximation error of NN-RBF and the uncertainties) that the adaptive term $\left(u_{i N}(t)\right)$ cannot compensate.

Taking into account these considerations, from (28), the control law that combines SMC and ANC is expressed as

$$
\begin{aligned}
& u_{i}(t)=\kappa_{i}\left[-K_{D i} S_{i}-f_{i}(\mathbf{x})+\dot{x}_{\text {refi }}-k_{i} e_{i}-\left(u_{i N}\right)\right. \\
& \left.-\Gamma_{i} \operatorname{sign}\left(S_{i}\right)\right],
\end{aligned}
$$

where $u_{i N}$ is a compensation variable which can be approximated by the neural function and it is the main structure of the ANC; the compensation variable can be represented as function of the optimal neural parameters, indicated by

$$
\begin{aligned}
u_{i N}^{*}= & \mathbf{v}_{i}^{* T} \boldsymbol{\varphi}^{*}\left(\zeta, \mathbf{c}^{*}, \boldsymbol{\rho}^{*}\right)+\sum_{j=1}^{2}\left(\mathbf{w}^{* T} \boldsymbol{\varphi}^{*}\left(\zeta, \mathbf{c}^{*}, \boldsymbol{\rho}^{*}\right)\right)_{i j} u_{j} \\
& +\varepsilon_{i n} \quad i=1,2
\end{aligned}
$$

where $\mathbf{v}^{*} \in \mathfrak{R}^{(n \times m)}, \mathbf{w}^{*} \in \mathfrak{R}^{(n \times m)}$, and $\boldsymbol{\varphi}^{*} \in \mathfrak{R}^{(m \times 1)}$ are optimal parameter vectors of the weights $\mathbf{v}$, the input weights vector $\mathbf{w}$, and the radial basis functions $\varphi$, respectively; $\mathbf{c}^{*}$ and $\boldsymbol{\rho}^{*}$ are the optimal values of the respective vectors (centers c and widths $\boldsymbol{\rho}$ ); and $\varepsilon_{n}$ is the neural approximation error generated by the ANC, and it is bounded by $\left|\varepsilon_{\text {Max }}\right| \geq\left|\varepsilon_{\text {in }}\right|>0$.

Other neural parameters of the ANC are defined by $\zeta$ and $m$ : the first one is the regressor vector and can be defined as $\zeta=\left[\mathbf{x}(t), \dot{\mathbf{x}}(t), \mathbf{x}_{\mathrm{ref}}^{k}(t), \mathbf{u}(t)\right]^{T}$ and the second one is the number of RBF neurons $(m=5)$ defined by empirical criterion [21]. The vector of $\operatorname{RBF}$ functions is $(\zeta)=$ $\left[\varphi_{1}(\zeta), \varphi_{2}(\zeta), \ldots, \varphi_{m}(\zeta)\right]^{T}$; each vector component is defined by

$$
\varphi_{i}^{*}\left(\boldsymbol{\zeta}, \mathbf{c}^{*}, \boldsymbol{\rho}^{*}\right)=\exp \left[-\rho_{i}^{* 2}\left\|\zeta-\mathbf{c}_{i}^{*}\right\|^{2}\right]
$$

with $\mathbf{c}^{*}=\left[\begin{array}{llll}c_{1}^{*} & c_{2}^{*} & \cdots & c_{n}^{*}\end{array}\right]^{T}$ in the center of the receptive field and $\rho^{*}$ is the width of the Gaussian function.

The optimal parameters of (36) are constant and unknown. These parameters are used as analytical tool to estimate their real values. The estimate neural parameters are $\widehat{\mathbf{v}}, \widehat{\mathbf{w}}$, and $\widehat{\boldsymbol{\varphi}}$, and they are estimated parameter vectors of $\mathbf{v}^{*}$, $\mathbf{w}^{*}$, and $\boldsymbol{\varphi}^{*}$, respectively; and $\widehat{\mathbf{c}}$ and $\widehat{\boldsymbol{\rho}}$ are the corresponding estimation of $\mathrm{c}^{*}$ and $\boldsymbol{\rho}^{*}$, respectively.

Defining the neural parameter approximation error (weights) like $\widetilde{\mathbf{v}}=\mathbf{v}^{*}-\widehat{\mathbf{v}}, \widetilde{\mathbf{w}}=\mathbf{w}^{*}-\widehat{\mathbf{w}}$ and function error 
like $\widetilde{\varphi}=\varphi^{*}-\widehat{\varphi}$, the neural compensation expressed in (36) can be written as

$$
\begin{aligned}
u_{i N}= & \widehat{\mathbf{v}}_{i}^{T} \widehat{\boldsymbol{\varphi}}(\zeta, \widehat{\mathbf{c}}, \widehat{\boldsymbol{\rho}})+\widetilde{\mathbf{v}}_{i}^{T} \widehat{\boldsymbol{\varphi}}(\zeta, \widehat{\mathbf{c}}, \widehat{\boldsymbol{\rho}})+\widehat{\mathbf{v}}_{i}^{T} \widetilde{\boldsymbol{\varphi}}(\zeta, \widetilde{\mathbf{c}}, \widetilde{\boldsymbol{\rho}}) \\
& +\widetilde{\mathbf{v}}_{i}^{T} \widetilde{\boldsymbol{\varphi}}(\zeta, \widetilde{\mathbf{c}}, \widetilde{\boldsymbol{\rho}})+\cdots+\sum_{j=1}^{2}\left(\widehat{\mathbf{w}}^{T} \widehat{\boldsymbol{\varphi}}(\zeta, \widehat{\mathbf{c}}, \widehat{\boldsymbol{\rho}})\right)_{i j} u_{j} \\
& +\sum_{j=1}^{2}\left(\widetilde{\mathbf{w}}^{T} \widehat{\boldsymbol{\varphi}}(\zeta, \widehat{\mathbf{c}}, \widehat{\boldsymbol{\rho}})\right)_{i j} u_{j} \\
& +\sum_{j=1}^{2}\left(\widehat{\mathbf{w}}^{T} \widetilde{\boldsymbol{\varphi}}(\zeta, \widetilde{\mathbf{c}}, \widetilde{\boldsymbol{\rho}})\right)_{i j} u_{j} \\
& +\sum_{j=1}^{2}\left(\widetilde{\mathbf{w}}^{T} \widetilde{\boldsymbol{\varphi}}(\zeta, \widetilde{\mathbf{c}}, \widetilde{\boldsymbol{\rho}})\right)_{i j} u_{j}+\varepsilon_{i n},
\end{aligned}
$$

where $\widetilde{\mathbf{v}}_{i}^{T} \widehat{\boldsymbol{\varphi}}+\widehat{\mathbf{v}}_{i}^{T} \widetilde{\boldsymbol{\varphi}}$ and $\widetilde{\mathbf{w}}^{T} \widehat{\boldsymbol{\varphi}}+\widehat{\mathbf{w}}^{T} \widetilde{\boldsymbol{\varphi}}$ represent the learning error of each neural term, and the terms (product of two errors) like $\widetilde{\mathbf{v}}_{i}^{T} \widetilde{\boldsymbol{\varphi}}$ and $\sum_{j=1}^{2}\left(\widetilde{\mathbf{w}}^{T} \widetilde{\boldsymbol{\varphi}}\right)_{i j} u_{j}$ can be considered into $\varepsilon_{i n}$.

Taking into account $[22,23]$, the neural approximations can uniformly approximate any continuous function. Now, it is possible to consider that $\widehat{\mathbf{v}}_{i} \widehat{\boldsymbol{\varphi}}=\Delta f_{i}(x)$ and $\sum_{j=1}^{2}\left(\widehat{\mathbf{w}}^{T} \widehat{\boldsymbol{\varphi}}\right)_{i j} u_{j}=$ $\sum_{j=1}^{2} \Delta g_{i j}(\mathbf{x}) u_{j}$

To obtain the closed loop equation, it is necessary to consider the proposed sliding surface (25), and replacing the robotic system (19) and also control law (35) with neural compensation (38), the closed loop equation becomes

$$
\begin{aligned}
\dot{S}_{i}= & -K_{D i} S_{i}-\widetilde{\mathbf{v}}_{i}^{T} \widehat{\boldsymbol{\varphi}}(\zeta, \widehat{\mathbf{c}}, \widehat{\boldsymbol{\rho}})-\widehat{\mathbf{v}}_{i}^{T} \widetilde{\boldsymbol{\varphi}}(\zeta, \widehat{\mathbf{c}}, \widehat{\boldsymbol{\rho}}) \\
& -\sum_{j=1}^{2}\left(\widetilde{\mathbf{w}}_{i}^{T} \widehat{\boldsymbol{\varphi}}(\zeta, \widehat{\mathbf{c}}, \widehat{\boldsymbol{\rho}})\right)_{i j} u_{j}-\cdots \\
& -\sum_{j=1}^{2}\left(\widehat{\mathbf{w}}_{i}^{T} \widetilde{\boldsymbol{\varphi}}(\zeta, \widehat{\mathbf{c}}, \widehat{\boldsymbol{\rho}})\right)_{i j} u_{j}-\varepsilon_{i n}-\Gamma_{i} \operatorname{sign}\left(S_{i}\right) .
\end{aligned}
$$

Using a Taylor's series to approximate the function difference $\widetilde{\boldsymbol{\varphi}}=\boldsymbol{\varphi}^{*}\left(\boldsymbol{\zeta}, \mathbf{c}^{*}, \boldsymbol{\rho}^{*}\right)-\widehat{\boldsymbol{\varphi}}(\boldsymbol{\zeta}, \widehat{\mathbf{c}}, \widehat{\boldsymbol{\rho}})$ and taking the point $\mathrm{c}^{*}=\widehat{\mathbf{c}}$ and $\boldsymbol{\rho}^{*}=\widehat{\boldsymbol{\rho}}$, the expansion can be described by

$$
\begin{aligned}
\boldsymbol{\varphi}^{*}\left(\boldsymbol{\zeta}, \mathbf{c}^{*}, \boldsymbol{\rho}^{*}\right)= & \widehat{\boldsymbol{\varphi}}(\boldsymbol{\zeta}, \widehat{\mathbf{c}}, \widehat{\boldsymbol{\rho}})+\Theta^{T} \widetilde{\mathbf{c}}+\Lambda^{T} \widetilde{\boldsymbol{\eta}} \\
& +\mathbf{O}(\zeta, \widetilde{\mathbf{c}}, \widetilde{\boldsymbol{\rho}}),
\end{aligned}
$$

where $\mathbf{O}(\cdot)$ represents the high-order term in a Taylor's series approximation and $\Theta$ and $\Lambda$ are derivatives of $\varphi^{*}\left(\zeta, c^{*}, \rho^{*}\right)$ with respect to $\mathbf{c}^{*}$ and $\boldsymbol{\rho}^{*}$ at $(\widehat{\mathbf{c}}, \widehat{\boldsymbol{\rho}})$. They are expressed as

$$
\begin{aligned}
& \boldsymbol{\Theta}^{T}=\left.\left(\frac{\partial \varphi\left(\zeta, c^{*}, \rho^{*}\right)}{\partial \mathbf{c}^{*}}\right)\right|_{\substack{\mathbf{c}^{*}=\widehat{c} \\
\boldsymbol{\rho}^{*}=\widehat{\rho}}}, \\
& \Lambda^{T}=\left.\left(\frac{\partial \varphi\left(\zeta, \mathbf{c}^{*}, \boldsymbol{\rho}^{*}\right)}{\partial \boldsymbol{\rho}^{*}}\right)\right|_{\substack{\mathbf{c}^{*}=\widehat{\mathbf{c}} \\
\boldsymbol{\rho}^{*}=\widehat{\boldsymbol{\rho}}}} .
\end{aligned}
$$

From (40), the following is obtained:

$$
\widetilde{\boldsymbol{\varphi}}=\boldsymbol{\Theta}^{T} \widetilde{\mathbf{c}}+\Lambda^{T} \widetilde{\boldsymbol{\eta}}+\mathbf{O}(\zeta, \widetilde{\mathbf{c}}, \widetilde{\boldsymbol{\rho}}) .
$$

From (42), the high-order term $\mathbf{O}(\cdot)$ is bounded by

$$
\begin{aligned}
\|\mathbf{O}(\zeta, \widetilde{\mathbf{c}}, \widetilde{\boldsymbol{\rho}})\| & =\left\|\widetilde{\boldsymbol{\varphi}}(\cdot)-\boldsymbol{\Theta}^{T} \widetilde{\mathbf{c}}-\Lambda^{T} \widetilde{\boldsymbol{\rho}}\right\| \leq \cdots \\
& \leq\|\widetilde{\boldsymbol{\varphi}}(\cdot)\|+\left\|\boldsymbol{\Theta}^{T} \widetilde{\mathbf{c}}\right\|+\left\|\boldsymbol{\Lambda}^{T} \widetilde{\boldsymbol{\rho}}\right\| \\
& \leq b_{1}+b_{2}\|\widetilde{\mathbf{c}}\|+b_{3}\|\tilde{\boldsymbol{\rho}}\| \leq \mathbf{O}_{\mathrm{Max}},
\end{aligned}
$$

where $b_{1}, b_{2}$, and $b_{3}$ are constants used to determine the boundedness of $\mathbf{O}(\cdot)$, due to the fact that the radial basis functions (gauss bell functions) and its derivatives are bounded by $b_{1}, b_{2}$, and $b_{3}$. Replacing (42) with (39), the following can be obtained:

$$
\begin{aligned}
\dot{S}_{i}= & -K_{D i} S_{i}-\widetilde{\mathbf{v}}_{i}^{T} \widehat{\boldsymbol{\varphi}}(\boldsymbol{\zeta}, \widehat{\mathbf{c}}, \widehat{\boldsymbol{\rho}})-\widehat{\mathbf{v}}_{i}^{T}\left(\boldsymbol{\Theta}^{T} \widetilde{\mathbf{c}}+\Lambda^{T} \widetilde{\boldsymbol{\rho}}+\mathbf{O}\right) \\
& -\sum_{j=1}^{2}\left(\widetilde{\mathbf{w}}^{T} \widehat{\boldsymbol{\varphi}}(\boldsymbol{\zeta}, \widehat{\mathbf{c}}, \widehat{\boldsymbol{\rho}})\right)_{i j} u_{j}-\cdots \\
& -\sum_{j=1}^{2}\left(\widehat{\boldsymbol{w}}^{T}\left(\boldsymbol{\Theta}^{T} \widetilde{\mathbf{c}}+\boldsymbol{\Lambda}^{T} \widetilde{\boldsymbol{\rho}}+\mathbf{O}\right)\right)_{i j} u_{j}-\varepsilon_{1 n} \\
& -\Gamma_{i} \operatorname{sign}\left(S_{i}\right) .
\end{aligned}
$$

Now, considering all terms that contain high-order terms $(\mathbf{O}(\cdot))$ in (44) and including the neural approximation error, they can be expressed as " $\varepsilon_{\mathrm{Max}}$ " " and it is bounded by a constant, as shown in (45):

$$
\left|\varepsilon_{\text {Max }}\right|=\left|\widehat{\mathbf{v}}_{i}^{T} \mathbf{O}+\sum_{j=1}^{2}\left(\widehat{\mathbf{w}}^{T} \mathbf{O}\right)_{i j} u_{j}+\varepsilon_{i n}\right| \leq \Gamma_{i} \quad i=1,2
$$

\section{Neural Parameters Tuning}

To achieve a stable adjustment law, a Lyapunov's candidate function must be proposed and it must consider all neural parameters (error, neural weights, spreads, and centers); the proposed function is

$$
V=\frac{1}{2}\left\{\sum_{i=1}^{2}\left[S_{\Omega i}^{2}+\frac{\widetilde{\mathbf{v}}_{i}^{T} \widetilde{\mathbf{v}}_{i}}{\alpha_{i}}+\frac{\widetilde{\mathbf{w}}_{i}^{T} \widetilde{\mathbf{w}}_{i}}{\beta_{i}}\right]+\left(\frac{\widetilde{\mathbf{c}}^{T} \widetilde{\mathbf{c}}}{\gamma_{1}}+\frac{\widetilde{\boldsymbol{\rho}}^{T} \widetilde{\boldsymbol{\rho}}}{\gamma_{2}}\right)\right\},
$$

where $\alpha_{i} \in \mathfrak{R}^{1 \times 1}, \beta_{i} \in \mathfrak{R}^{1 \times 1}, \gamma_{1} \in \mathfrak{R}^{1 \times 1}$, and $\gamma_{2} \in \mathfrak{R}^{1 \times 1}$ are nonnegative constants, respectively. $n$ is outputs number $(n=2)$ and $m$ is neurons number $(m=5)$. In this analysis, to reduce any possible cause of chattering effect, a boundary layer thickness $\Omega_{i}$ is defined, and $S_{\Omega i}$ can be computed as follows:

$$
\begin{gathered}
S_{\Omega i}=S_{i}-\Omega_{i} \operatorname{sat}\left(\frac{S_{i}}{\Omega_{i}}\right) \\
\operatorname{sat}\left(\frac{S_{i}}{\Omega_{i}}\right)= \begin{cases}\operatorname{sign}\left(S_{i}\right) & \text { if }\left|S_{i}\right| \geq \Omega_{i} \\
\left(\frac{S_{i}}{\Omega_{i}}\right) & \text { if }\left|S_{i}\right|<\Omega_{i} .\end{cases}
\end{gathered}
$$


In this analysis, the sign function was changed by a saturation function, in similar way as done by $[14,24]$. This boundary layer (BL) smoothes out the control behavior and ensures that the system states remain within this layer

$$
S_{\Omega i}=\left\{e_{i} \in \mathbb{R}\left|S_{i}\left(e_{i}\right) \leq\right| \Omega_{i} \mid\right\} .
$$

Now, the derivative of $V$ (46) with respect to time is written in (49), noting that $S_{\Omega i}=0$ inside the BL and $\dot{S}_{\Omega i}=\dot{S}_{i}$ outside. Then (49) can be expressed as

$$
\begin{aligned}
\frac{d V}{d t}= & \sum_{i=1}^{2}\left[S_{\Omega i} \frac{d S_{i}}{d t}+\alpha_{i}^{-1} \widetilde{\mathbf{v}}_{i}^{T} \frac{d \widetilde{\mathbf{v}}_{i}}{d t}+\beta_{i}^{-1} \widetilde{\mathbf{w}}_{i}^{T} \frac{d \widetilde{\mathbf{w}}_{i}}{d t}\right] \\
& +\left(\gamma_{1}^{-1} \frac{d \widetilde{\mathbf{c}}^{T}}{d t} \widetilde{\mathbf{c}}+\gamma_{2}^{-1} \frac{d \widetilde{\boldsymbol{\rho}}^{T}}{d t} \widetilde{\boldsymbol{\rho}}\right) .
\end{aligned}
$$

Substituting (44) in (49), the following is obtained:

$$
\begin{aligned}
& \frac{d V}{d t}=\sum_{i=1}^{2}\left[-K_{D i} S_{\Omega i}^{2}-S_{\Omega i} \widetilde{\mathbf{v}}_{i}^{T} \widehat{\boldsymbol{\varphi}}(\boldsymbol{\zeta}, \widehat{\mathbf{c}}, \widehat{\boldsymbol{\rho}})-S_{\Omega i} \widehat{\mathbf{v}}_{i}^{T} \boldsymbol{\Theta}^{T} \widetilde{\mathbf{c}}\right. \\
& \quad-S_{\Omega i} \widehat{\mathbf{v}}_{i}^{T} \boldsymbol{\Lambda}^{T} \widetilde{\boldsymbol{\rho}}-S_{\Omega i} \widehat{\mathbf{v}}_{i}^{T} \mathbf{O} \\
& \quad-S_{\Omega i} \sum_{j=1}^{2}\left(\widetilde{\mathbf{w}}^{T} \widehat{\boldsymbol{\varphi}}(\boldsymbol{\zeta}, \widehat{\mathbf{c}}, \widehat{\boldsymbol{\rho}})\right)_{i j} u_{j}-\cdots \\
& \quad-S_{\Omega i} \sum_{j=1}^{2}\left(\widehat{\mathbf{w}}_{i}^{T}\left(\boldsymbol{\Theta}^{T} \widetilde{\mathbf{c}}+\boldsymbol{\Lambda}^{T} \widetilde{\boldsymbol{\rho}}+\mathbf{O}\right)\right)_{i j} u_{j}-S_{\Omega i} \varepsilon_{i n} \\
& \left.\quad-\Gamma_{i}\left|S_{\Omega i}\right|+\alpha_{i}^{-1} \widetilde{\mathbf{v}}_{i}^{T} \frac{d \widetilde{\mathbf{v}}_{i}}{d t}+\beta_{i}^{-1} \widetilde{\mathbf{w}}_{i}^{T} \frac{d \widetilde{\mathbf{w}}_{i}}{d t}\right]+\cdots \\
& +\left(\gamma_{1}^{-1} \frac{d \widetilde{\mathbf{c}}^{T}}{d t} \widetilde{\mathbf{c}}+\gamma_{2}^{-1} \frac{d \widetilde{\boldsymbol{\rho}}^{T}}{d t} \widetilde{\boldsymbol{\rho}}\right) .
\end{aligned}
$$

Rearranging (50),

$$
\begin{aligned}
& \frac{d V}{d t}=\sum_{i}\left[-K_{D i} S_{\Omega i}^{2}-S_{\Omega i} \widetilde{\mathbf{v}}_{i}^{T} \widehat{\boldsymbol{\varphi}}(\boldsymbol{\zeta}, \widehat{\mathbf{c}}, \widehat{\boldsymbol{\rho}})-S_{\Omega i} \widehat{\mathbf{v}}_{i}^{T} \boldsymbol{\Theta}^{T} \widetilde{\mathbf{c}}\right. \\
& -S_{\Omega i} \widehat{\mathbf{v}}_{i}^{T} \boldsymbol{\Lambda}^{T} \widetilde{\boldsymbol{\rho}}-S_{\Omega i} \widehat{\mathbf{v}}_{i}^{T} \mathbf{O}+\alpha_{i}^{-1} \widetilde{\mathbf{v}}_{i}^{T} \frac{d \widetilde{\mathbf{v}}_{i}}{d t}+\beta_{i}^{-1} \widetilde{\mathbf{w}}_{i}^{T} \frac{d \widetilde{\mathbf{w}}_{i}}{d t} \\
& -S_{\Omega i} \sum_{j=1}^{2}\left(\widehat{\mathbf{w}}_{i}^{T} \boldsymbol{\Theta}^{T} \widetilde{\mathbf{c}}\right)_{i j} u_{j}-S_{\Omega i} \sum_{j=1}^{2}\left(\widehat{\mathbf{w}}_{i}^{T} \boldsymbol{\Lambda}^{T} \widetilde{\boldsymbol{\rho}}\right)_{i j} u_{j} \\
& -S_{\Omega i} \sum_{j=1}^{2}\left(\widehat{\mathbf{w}}_{i}^{\mathrm{T}} \mathbf{O}\right)_{i j} u_{j}-S_{\Omega i} \sum_{j=1}^{2}\left(\widetilde{\mathbf{w}}^{T} \widehat{\boldsymbol{\varphi}}(\boldsymbol{\zeta}, \widehat{\mathbf{c}}, \widehat{\boldsymbol{\rho}})\right)_{i j} u_{j} \\
& \left.-S_{\Omega i} \varepsilon_{i n}-\Gamma_{i}\left|S_{\Omega i}\right|\right]+\cdots+\left(\gamma_{1}^{-1} \frac{d \widetilde{\mathbf{c}}^{T}}{d t} \widetilde{\mathbf{c}}+\gamma_{2}^{-1} \frac{d \widetilde{\boldsymbol{\rho}}^{T}}{d t}\right. \\
& \cdot \widetilde{\boldsymbol{\rho}}) .
\end{aligned}
$$

Now grouping terms in (51) leads to

$$
\begin{aligned}
\frac{d V}{d t} & =\sum_{i=1}^{2}\left[-K_{D i} S_{\Omega i}^{2}+\widetilde{\mathbf{v}}_{i}^{T}\left(-S_{\Omega i} \widehat{\boldsymbol{\varphi}}(\boldsymbol{\zeta}, \widehat{\mathbf{c}}, \widehat{\boldsymbol{\rho}})+\alpha_{i}^{-1} \frac{d \widetilde{\mathbf{v}}_{i}}{d t}\right)\right. \\
& +\widetilde{\mathbf{w}}_{i}^{T}\left(-S_{\Omega i} \sum_{j=1}^{2} \widehat{\boldsymbol{\varphi}}_{j}(\boldsymbol{\zeta}, \widehat{\mathbf{c}}, \widehat{\boldsymbol{\rho}}) u_{j}+\beta_{i}^{-1} \frac{d \widetilde{\mathbf{w}}_{i}}{d t}\right)-\cdots \\
& \left.-S_{\Omega i}\left(\widehat{\mathbf{v}}_{i}^{T} \mathbf{O}+\sum_{j=1}^{2}\left(\widehat{\mathbf{w}}^{T} \mathbf{O}\right)_{i j} u_{j}+\varepsilon_{i n}\right)-\Gamma_{i}\left|S_{\Omega i}\right|\right] \\
& +\left(-\sum_{i=1}^{2}\left[S_{\Omega i} \widehat{\mathbf{v}}_{i}^{T} \boldsymbol{\Lambda}^{T}+S_{\Omega i} \sum_{j=1}^{2}\left(\widehat{\mathbf{w}}^{T} \boldsymbol{\Lambda}^{T}\right)_{i j} u_{j}\right]\right.
\end{aligned}
$$

$$
\begin{aligned}
& \left.+\gamma_{2}^{-1} \frac{d \widetilde{\boldsymbol{\rho}}^{T}}{d t}\right) \tilde{\boldsymbol{\rho}}+\cdots \\
& +\left(-\sum_{i=1}^{2}\left[S_{\Omega i} \widehat{\mathbf{v}}_{i}^{T} \boldsymbol{\Theta}^{T}+S_{\Omega i} \sum_{j=1}^{2}\left(\widehat{\mathbf{w}}^{T} \boldsymbol{\Theta}^{T}\right)_{i j} u_{j}\right]\right. \\
& \left.+\gamma_{1}^{-1} \frac{d \widetilde{\mathbf{c}}^{T}}{d t}\right) \widetilde{\mathbf{c}} .
\end{aligned}
$$

Now $\dot{\widetilde{\mathbf{v}}}, \dot{\widetilde{\mathbf{w}}}, \dot{\widetilde{\mathbf{c}}}$, and $\dot{\widetilde{\boldsymbol{\rho}}}$ are selected as

$$
\begin{aligned}
& \frac{d \widetilde{\mathbf{v}}_{i}}{d t}=\alpha_{i} S_{\Omega i} \widehat{\boldsymbol{\varphi}}(\boldsymbol{\zeta}, \widehat{\mathbf{c}}, \widehat{\boldsymbol{\rho}}) \\
& \frac{d \widetilde{\mathbf{w}}_{i}}{d t}=\beta_{i} S_{\Omega i} \sum_{j=1}^{2} \widehat{\boldsymbol{\varphi}}_{j}(\boldsymbol{\zeta}, \widehat{\mathbf{c}}, \widehat{\boldsymbol{\rho}}) u_{j}, \\
& \frac{d \widetilde{\boldsymbol{\rho}}^{T}}{d t}=\gamma_{2} \sum_{i=1}^{2} S_{\Omega i}\left[\widehat{\mathbf{v}}_{i}^{T} \boldsymbol{\Lambda}^{T}+\sum_{j=1}^{2}\left(\widehat{\mathbf{w}}^{T} \boldsymbol{\Lambda}^{T}\right)_{i j} u_{j}\right] \\
& \frac{d \widetilde{\mathbf{c}}^{T}}{d t}=\gamma_{1} \sum_{i=1}^{2} S_{\Omega i}\left[\widehat{\mathbf{v}}_{i}^{T} \boldsymbol{\Theta}^{T}+\sum_{j=1}^{2}\left(\widehat{\mathbf{w}}^{T} \boldsymbol{\Theta}^{T}\right)_{i j} u_{j}\right] .
\end{aligned}
$$

Considering (53) into (52), then (52) can be rewritten as

$$
\begin{aligned}
\frac{d V}{d t} & =\sum_{i=1}^{2}-K_{D i} S_{\Omega i}^{2}-\Gamma_{i}\left|S_{\Omega i}\right| \\
& -S_{\Omega i}\left(\widehat{\mathbf{v}}_{i}^{T} \mathbf{O}+\sum_{j=1}^{2}\left(\widehat{\mathbf{w}}^{T} \mathbf{O}\right)_{i j} u_{j}+\varepsilon_{i n}\right) .
\end{aligned}
$$

From (54), it follows that

$$
\frac{d V}{d t} \leq \sum_{i}\left(-K_{D i} S_{i}^{2}+\left|\varepsilon_{\operatorname{Max}}\right|\left|S_{\Omega i}\right|-\Gamma_{i}\left|S_{\Omega i}\right|\right)<0 .
$$


TABLE 1: Mobile robot parameters.

\begin{tabular}{lccc}
\hline Parameters & Pioneer 3DX & Pioneer 2DX & Units \\
\hline$\kappa_{1}$ & 0.24089 & 0.3037 & $\mathrm{~s}$ \\
$\kappa_{2}$ & 0.2424 & 0.2768 & $\mathrm{~s}$ \\
$\kappa_{3}$ & $-9.3603 e^{-4}$ & $-4.018 e^{-4}$ & $\mathrm{~s} \cdot \mathrm{m} / \mathrm{rad}^{2}$ \\
$\kappa_{4}$ & 0.99629 & 0.9835 & \\
$\kappa_{5}$ & $-3.725 e^{-3}$ & $-3.818 e^{-3}$ & $\mathrm{~s} / \mathrm{m}$ \\
$\kappa_{6}$ & 1.0915 & 1.0725 & \\
\hline
\end{tabular}

We integrate both sides of (55) which is defined as

$$
\begin{gathered}
\int_{0}^{t_{S}} \sum_{i}\left(K_{D i}\left|S_{\Omega i}\right|^{2}+\Gamma_{i}\left|S_{\Omega i}\right|-\left|\varepsilon_{\operatorname{Max}}\right|\left|S_{\Omega i}\right|\right) d t \\
\leq-\int_{0}^{t_{S}}\left(\frac{d V}{d t}\right) d t=\left[V(0)-V\left(t_{s}\right)\right] .
\end{gathered}
$$

Because $V(0)$ is bounded, and $V\left(t_{s}\right)$ is also bounded and nonincreasing, the following can be obtained:

$$
\lim _{t_{S} \rightarrow \infty}\left[-\int_{0}^{t_{S}}\left(\frac{d V}{d t}\right) d t\right]=V(0)-V\left(t_{s}\right)<\infty .
$$

Therefore, by Barbalat's lemma [17], it can be shown that $\lim _{t_{S} \rightarrow \infty}(-d V(t) / d t)=0$. That is, $S(t)$ tends to zero as $t$ tends to infinity.

As a result, the proposed control is stable. Moreover, the control error of the dynamic part will converge to zero asymptotically according to $S(t) \rightarrow 0$.

From (53), considering $\dot{\mathbf{v}}_{i}^{*}=0, \dot{\mathbf{w}}_{i}^{*}=0, \dot{\mathbf{c}}^{*}=0$, and $\dot{\boldsymbol{\rho}}^{*}=$ 0 , the neural adjusting rules are

$$
\begin{aligned}
\frac{d \widehat{\mathbf{v}}_{i}}{d t} & =-\alpha_{i} S_{\Omega i} \widehat{\boldsymbol{\varphi}}(\boldsymbol{\zeta}, \widehat{\mathbf{c}}, \widehat{\boldsymbol{\rho}}), \\
\frac{d \widehat{\mathbf{w}}_{i}}{d t} & =-\beta_{i} S_{\Omega i} \sum_{j=1}^{2} \widehat{\boldsymbol{\varphi}}_{j}(\boldsymbol{\zeta}, \widehat{\mathbf{c}}, \widehat{\boldsymbol{\rho}}) u_{j}, \\
\frac{d \widetilde{\boldsymbol{\rho}}^{T}}{d t} & =-\gamma_{2}^{-1} \sum_{i=1}^{2} S_{\Omega i}\left[\widehat{\mathbf{v}}_{i}^{T} \boldsymbol{\Lambda}^{T}+\sum_{j=1}^{2}\left(\widehat{\mathbf{w}}^{T} \boldsymbol{\Lambda}^{T}\right)_{i j} u_{j}\right], \\
\frac{d \widehat{\mathbf{c}}^{T}}{d t} & =-\gamma_{1}^{-1} \sum_{i=1}^{2} S_{\Omega i}\left[\widehat{\mathbf{v}}_{i}^{T} \boldsymbol{\Theta}^{T}+\sum_{j=1}^{2}\left(\widehat{\mathbf{w}}_{i}^{T} \boldsymbol{\Theta}^{T}\right)_{i j} u_{j}\right] .
\end{aligned}
$$

Equations (58)-(61) are addressed to the tuning online of the dynamic compensation. Equations (58) and (59) adjust the neural weights, and (60) and (61) are responsible for setting the widths and the centers of each RBF vector of the ANC.

\section{Experimental Results and Discussion}

This section analyzes the performance of the proposed control technique, a single experiment is executed, and the corresponding results are presented below. To implement the experiment, a pioneer 2DX is used. The robot's parameters are indicated in Table 1. The control action commands

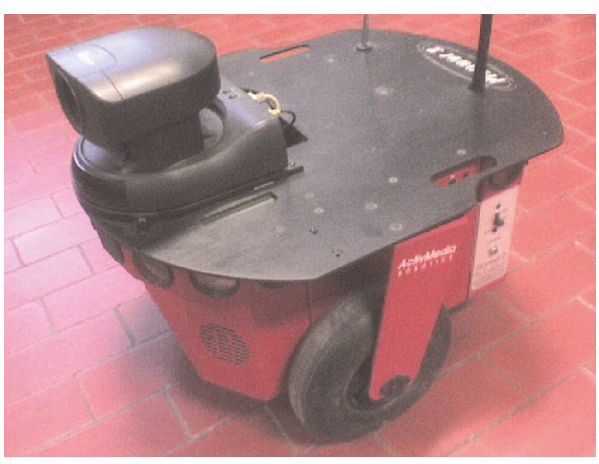

Figure 3: Pioneer 2DX mobile robot.

of the WMR are linear and angular velocities. Figure 3 shows a pioneer 2DX, and it has an $800 \mathrm{MHz}$ Intel-Pentium with $512 \mathrm{Mb}$ of memory RAM onboard CPU in which the proposed control technique is implemented.

In the experiment, the odometric sensors are used to sense the robot position; this experiment tries to demonstrate the advantages of CSMC-AMC method with respect to other control techniques from the literature.

The experiment is performed in the following manner: the robot must follow a predetermined trajectory (in this case a pentagon), where the dynamic controller is calibrated with different parameters not corresponding to the pioneer 2DX robot on the nominal model part $\left(f_{i}(x)\right.$ and $g_{i}(x)$ in $\left.(33)\right)$ (being the parameters of the 3DX pioneer robot).

The ANC (adaptive neural compensator) is activated at $50 \mathrm{sec}$. after initiating the experiment. Such compensator reduces the control error produced by the difference between the real and nominal dynamical structure, while the sliding part is used to eliminate the residual error added by the neural approximation, reducing the control error $e(t)$, and therefore the error position $e_{r}(t)$ too. The registered results in the experiment are presented in Figures 4-9.

The response driven by the proposed control technique shows a larger value of the trajectory error because the robot is far away from the reference trajectory in the initial moment. Since the ANC can compensate these phenomena through learning, it can be seen that after 50 secs. of the initial time, there is a transitory error due to the initial commutation of the ANC, but then the tracking errors are continuously reduced and a better tracking performance of the proposed control technique is obtained. Figure 6 shows the control actions and the WMR output speeds of the CSMC-ANC technique, while Figure 5 depicts the desired and followed trajectory by the WMR with CSMC-ANC.

In Figure 7, the trajectory errors for the experiments using the proposed CSMC-ANC technique to follow the desired reference trajectory are shown. The trajectory error is defined as the error norm of the difference between the instantaneous distance of the WMR and the desired reference. It does easily verify that the error at final time is less than the initial position error. Figures 8 and 9 show the neural weights evolution during the experiment. In both figures, it can be observed that the network starts to set the weights from $50 \mathrm{sec}$., that is, the instant when the ANC compensator is activated. 

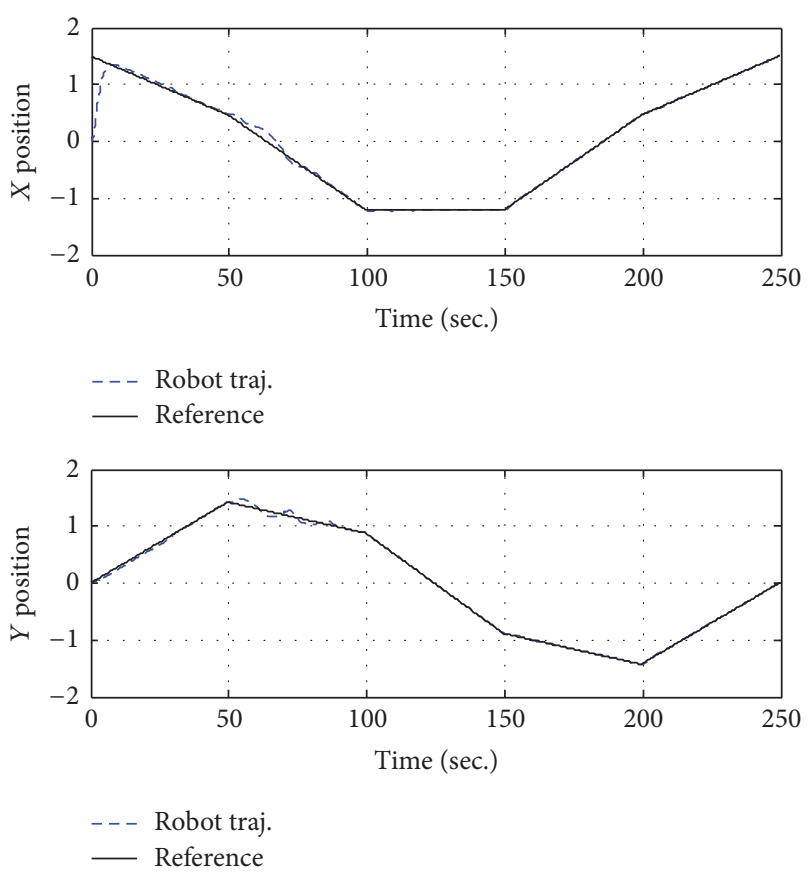

Figure 4: Reference signals of position and instantaneous mobile robot position.

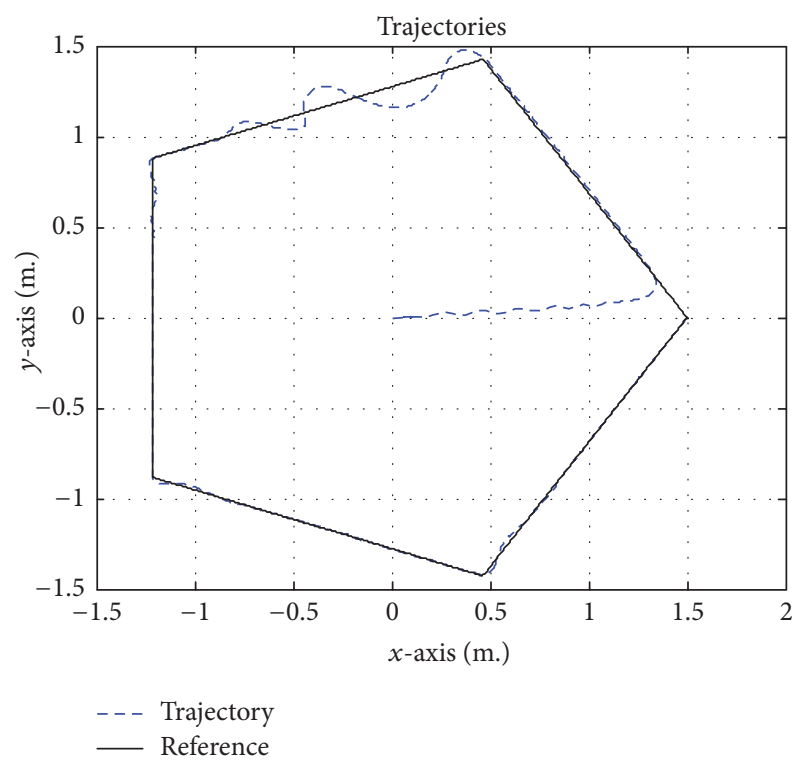

FIGURE 5: Desired and real trajectory followed by the WMR.

In this work, a control technique is presented that uses two SMC based controllers to control the trajectory of a mobile robot. The design of the proposed control technique was done to avoid the chattering effect. Therefore, the controller uses an adaptive part (ANC) to reduce the effect produced by the discontinuous function in the control action; this discontinuity is the main cause of this effect. And to ensure the reduction, the discontinuous function was replaced by the saturation function equation (47) smoothing the output response. Further, the cascade SMC with ANC is robust and efficient with respect to the dynamical variations
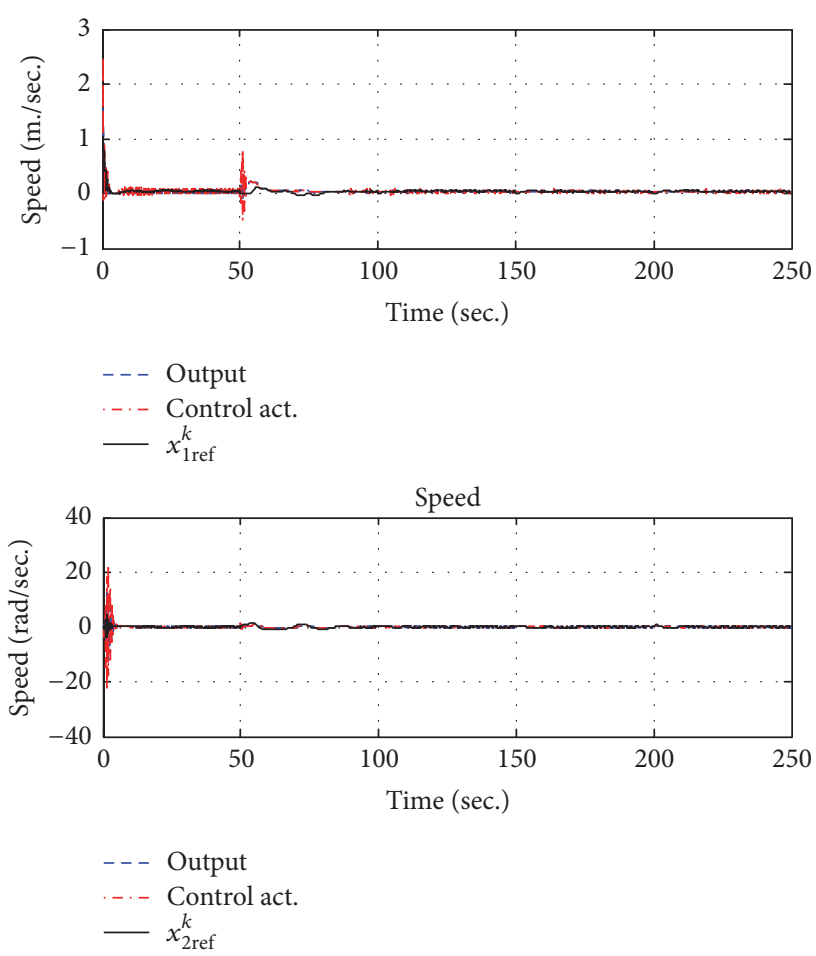

FIGURE 6: Control actions and output speeds of the WMR and by the cascade SMC with ANC.

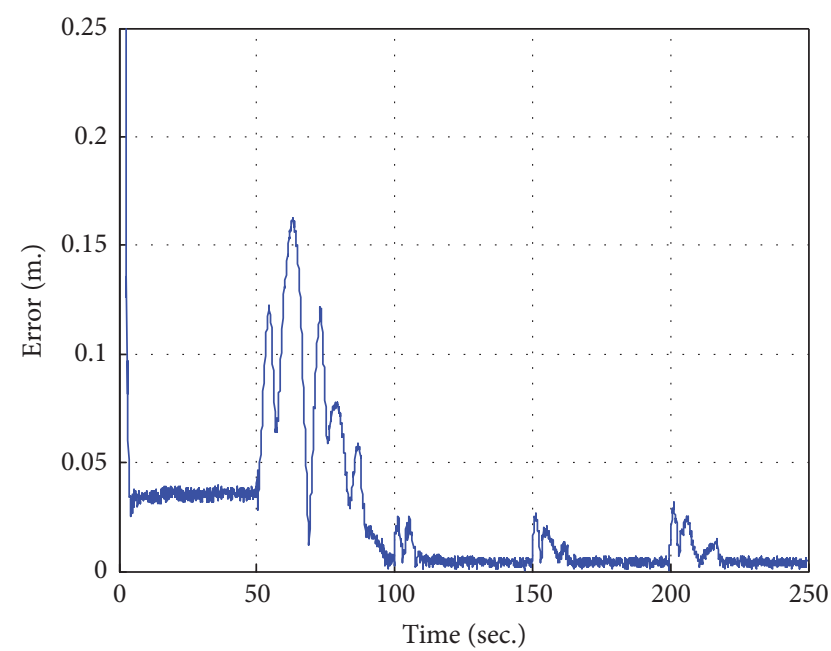

FIgURE 7: Trajectory error with cascade SMC and ANC activated after $50 \mathrm{sec}$.

of the WMR. In addition, it is effective in reducing the control error produced by the parameter variations or model uncertainties. The proposed controller designed in this research work for a WMR does not use the real dynamics for its implementation.

In the literature, there are many mathematical formulations for WMR dynamics not concerned with perfect accuracy, but the response of the cascade SMC-ANC as a control action to these dynamic variations as well as possible parameter variations of the WMR improves the system robustness. 

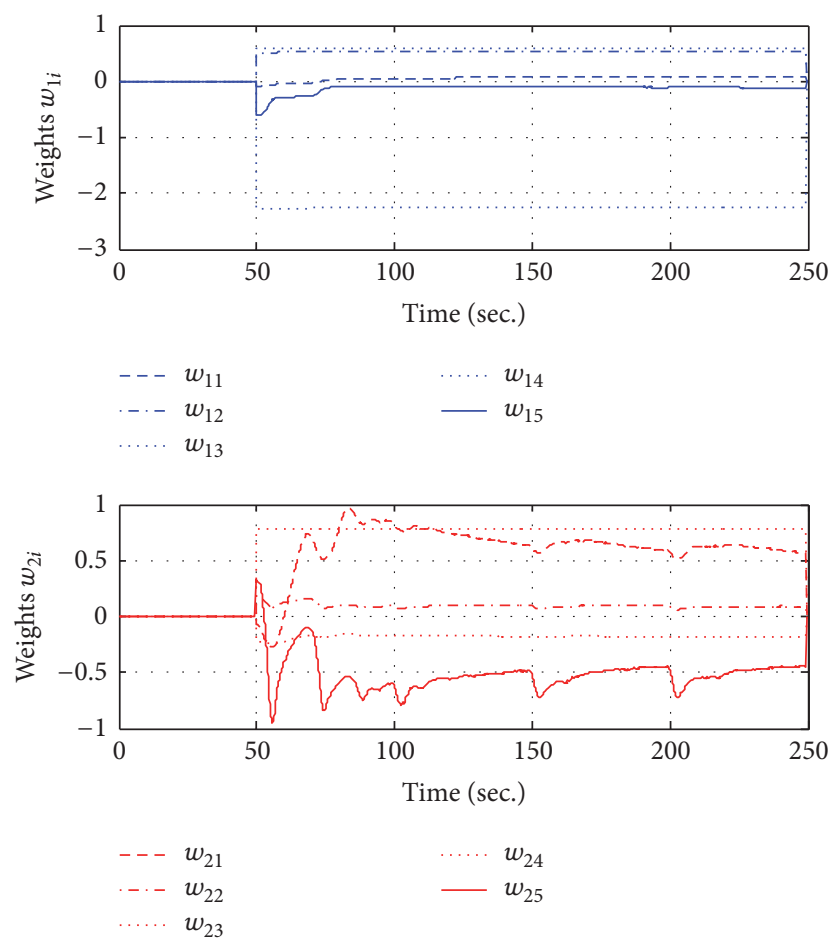

FIGURE 8: Evolution of the neural output weights $(w)$ of the cascade SMC with ANC during the experiment.

The proposed control technique design methodology presented in this research work admits two significant characteristics, with important implications for WMR control design applications. The first one, the pair CSMC-ANC, is stable and its control error tends to zero (Section 6). And second one can be considered as a possible solution for the control of WMR and of any nonlinear systems, when the uncertainties are present in the robot dynamics or there are parameter variations in the robot.

\section{Conclusions}

In this research work, the problem of using two SMC controllers connected in cascaded has been considered. One of them uses an adaptive neural compensation (ANC). This technique was applied to nonlinear systems with nonmodeled uncertainties.

In the proposed technique, it is necessary to highlight that the controller design of the dynamic part was built using the combination of a feedback linearization controller and a SMC with an adaptive neural compensation (ANC). This control technique guarantees asymptotic convergence through a Lyapunov-based adaptive neural compensation.

The ANC in combination with SMC can reduce the control error caused by parameters variations of the WMR that affects the feedback linearization built with the known dynamics. This compensation modifies the control action to reduce the effects of dynamic variations of the WMR or model uncertainties. The main advantage of this technique is that the ANC does not need to learn all the dynamics
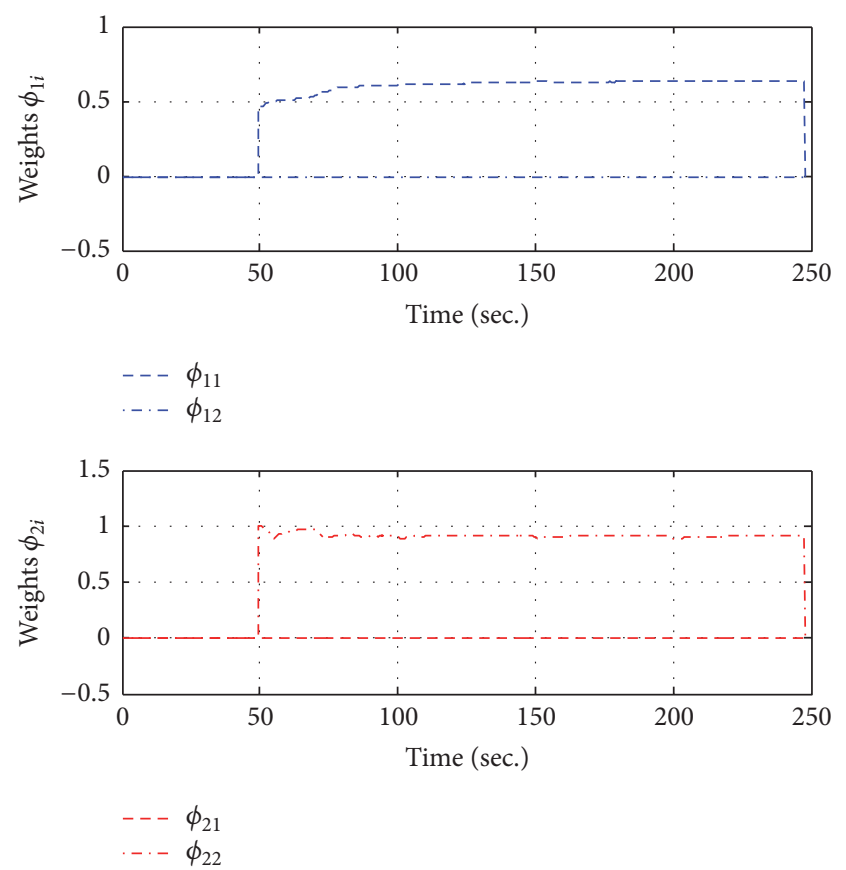

FIGURE 9: Evolution of the neural input weights $(\varphi)$ of the cascade SMC with ANC during the experiment.

of the system structure, and it was designed to compensate only the dynamic variations and all possible uncertainties in the model. All other errors tend to zero by the action of the CSMC.

By experimental results, the proposed method has been shown to reduce trajectory errors more than the conventional SMC.

\section{Appendix}

\section{Parameters Description}

The identified parameters [15] can be described by

$$
\begin{aligned}
& \kappa_{1}=\left(\frac{\left(\left(R_{a} / k_{a}\right)\left(M R_{t} r+2 I_{e}\right)+2 r k_{D T}\right)}{2 r k_{P T}}\right), \\
& \kappa_{2} \\
& =\left(\frac{\left(\left(R_{a} / k_{a}\right)\left(I_{e} d^{2}+2 R_{t} r\left(I_{z}+M b^{2}\right)\right)+2 r d k_{D R}\right)}{2 r d k_{P R}}\right), \\
& \kappa_{3}=\left(\frac{\left(R_{a} / k_{a}\right) M b R_{t}}{2 k_{P T}}\right) ; \\
& \kappa_{4}=\left(\frac{\left(R_{a} / k_{a}\right)\left(k_{a} k_{b} / R_{a}+B_{e}\right)}{r k_{P T}}+1\right), \\
& \kappa_{5}=\left(\frac{\left(R_{a} / k_{a}\right) M b R_{t}}{d k_{P R}}\right) ; \\
& \kappa_{6}=\left(\frac{\left(R_{a} / k_{a}\right)\left(k_{a} k_{b} / R_{a}+B_{e}\right) d}{2 r k_{P R}}+1\right) .
\end{aligned}
$$


In these relations, $M$ is the robot mass; $r$ is the radius of the left and right wheels; $k_{b}$ is equal to the electromotoric force constant multiplied by the reduction constant; $R_{a}$ is the electric resistance; $k_{a}$ is the constant of torque multiplied by the reduction constant; $k_{P R}, k_{P T}, y k_{D T}$ are positive constants; $I_{e}$ and $B_{e}$ are the moment of inertia and the viscous friction coefficient both belonging to the combination of motor, gearbox, and wheel; and $R_{t}$ is the nominal radius of the wheel.

\section{Conflicts of Interest}

The authors declare that there are no conflicts of interest regarding the publication of this paper.

\section{Acknowledgments}

This work was funded by the National Council of Scientific and Technological Research (CONICET) PIO-CONICET Project no. 15020150100084 CO.

\section{References}

[1] K.-S. Huang, Y.-H. Lin, K.-B. Lin, B.-K. Lee, and C.-K. Hwang, "Cascade sliding mode control of a spherical wheel robot driven by Omni wheels," in Proceedings of the International Conference on Machine Learning and Cybernetics (ICMLC '12), pp. 16071611, Xian, China, July 2012.

[2] S. Peng and W. Shi, "Adaptive fuzzy integral terminal sliding mode control of a nonholonomic wheeled mobile robot," Mathematical Problems in Engineering, vol. 2017, Article ID 3671846, 12 pages, 2017.

[3] H. Ashrafiuon, S. Nersesov, and G. Clayton, "Trajectory tracking control of planar underactuated vehicles," IEEE Transactions on Automatic Control, vol. 62, no. 4, pp. 1959-1965, 2017.

[4] P. Proaño, L. Capito, G. Scaglia, A. Rosales, and O. Camacho, "Comparison of two linear algebra approaches for mobile robots controllers design," International Journal of Automation and Control, 2016.

[5] J. Mu, X. G. Yan, S. K. Spurgeon, and Z. Mao, "Generalised regular form based SMC for nonlinear systems with application to a WMR," IEEE Transactions on Industrial Electronics, vol. 64, no. 8, pp. 6714-6723, 2017.

[6] W. Sun, S. Tang, H. Gao, and J. Zhao, "Two time-scale tracking control of nonholonomic wheeled mobile robots," IEEE Transactions on Control Systems Technology, vol. 24, no. 6, pp. 20592069, 2016.

[7] H. Guo, Y. Liu, G. Liu, and H. Li, "Cascade control of a hydraulically driven 6-DOF parallel robot manipulator based on a sliding mode," Control Engineering Practice, vol. 16, no. 9, pp. 1055-1068, 2008.

[8] F. G. Rossomando, C. Soria, and R. Carelli, "Neural networkbased compensation control of mobile robots with partially known structure," IET Control Theory and Applications, vol. 6, no. 12, pp. 1851-1860, 2012.

[9] S. Luo, S. Wu, Z. Liu, and H. Guan, "Wheeled mobile robot RBFNN dynamic surface control based on disturbance observer," ISRN Applied Mathematics, vol. 2014, Article ID 634936, 9 pages, 2014.

[10] H. Mehrjerdi, Y. M. Zhang, and M. Saad, "Adaptive exponential sliding mode control for dynamic tracking of a nonholonomic mobile robot," Intelligent Robotics and Applications, vol. 7506, no. 1, pp. 643-652, 2012.

[11] V. Parra-Vega and G. Hirzinger, "Chattering-free sliding mode control for a class of nonlinear mechanical systems," International Journal of Robust and Nonlinear Control, vol. 11, no. 12, pp. 1161-1178, 2001.

[12] A. Bessas, A. Benalia, and F. Boudjema, "Integral sliding mode control for trajectory tracking of wheeled mobile robot in presence of uncertainties," Journal of Control Science and Engineering, vol. 2016, Article ID 7915375, 10 pages, 2016.

[13] F. G. Rossomando, C. Soria, and R. Carelli, "Autonomous mobile robots navigation using RBF neural compensator," Control Engineering Practice, vol. 19, no. 3, pp. 215-222, 2011.

[14] F. G. Rossomando, C. Soria, and R. Carelli, "Sliding mode neuro adaptive control in trajectory tracking for mobile robots," Journal of Intelligent Robotic Systems, vol. 74, no. 3, pp. 931-944, 2014.

[15] C. De La Cruz and R. Carelli, "Dynamic modeling and centralized formation control of mobile robots," in Proceedings of the 32nd Annual Conference on IEEE Industrial Electronics (IECON '06), pp. 3880-3885, Paris, France, November 2006.

[16] F. Valero, F. Rubio, C. Llopis-Albert, and J. I. Cuadrado, "Influence of the friction coefficient on the trajectory performance for a car-like robot," Mathematical Problems in Engineering, vol. 2017, Article ID 4562647, 9 pages, 2017.

[17] J.-J. E. Slotine and W. Li, Applied Nonlinear Control, vol. 199, Prentice-Hall, Englewood Cliffs, NJ, USA, 1991.

[18] C. Edwards and S. Spurgeon, Sliding Mode Control: Theory and Applications, CRC Press, 1998.

[19] V. I. Utkin, Sliding Modes in Control Optimization, Springer, Berlin, Germany, 2013.

[20] C. H. Tsai, H. Y. Chung, and F. M. Yu, "Neuro-sliding mode control with its applications to seesaw systems," IEEE Transactions on Neural Networks, vol. 15, no. 1, pp. 124-134, 2004.

[21] K. G. Sheela and S. N. Deepa, "Review on methods to fix number of hidden neurons in neural networks," Mathematical Problems in Engineering, vol. 2013, Article ID 425740, 11 pages, 2013.

[22] J. Park and I. W. Sandberg, "Universal approximation using radial-basis-function networks," Neural Computation, vol. 3, no. 2, pp. 246-257, 1991.

[23] G. Cybenko, "Approximation by superpositions of a sigmoidal function," Mathematics of Control, Signals, and Systems, vol. 2, no. 4, pp. 303-314, 1989.

[24] F. G. Rossomando, C. Soria, and R. Carelli, "Adaptive neural sliding mode compensator for a class of nonlinear systems with unmodeled uncertainties," Engineering Applications of Artificial Intelligence, vol. 26, no. 10, pp. 2251-2259, 2013. 


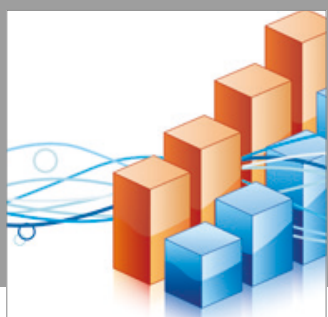

Advances in

Operations Research

vatersals

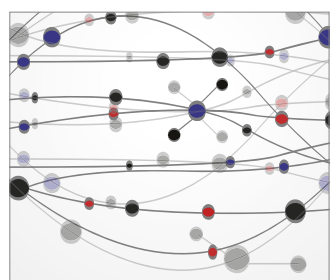

\section{The Scientific} World Journal
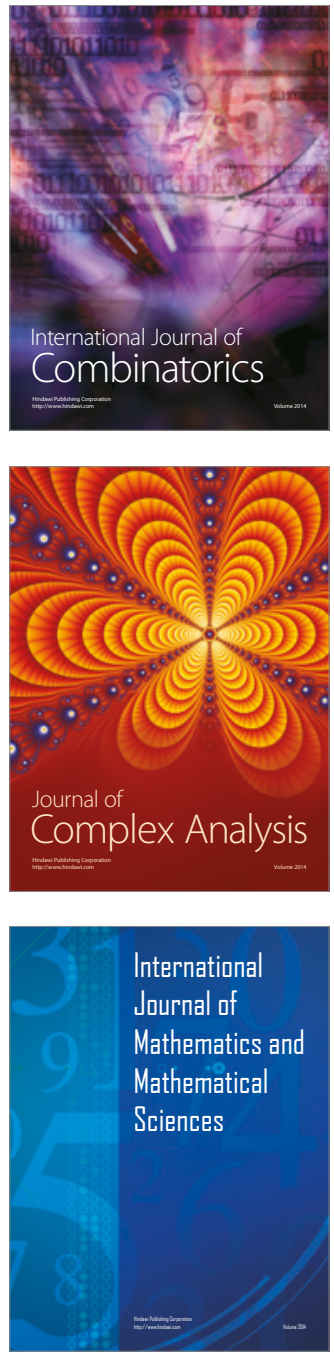
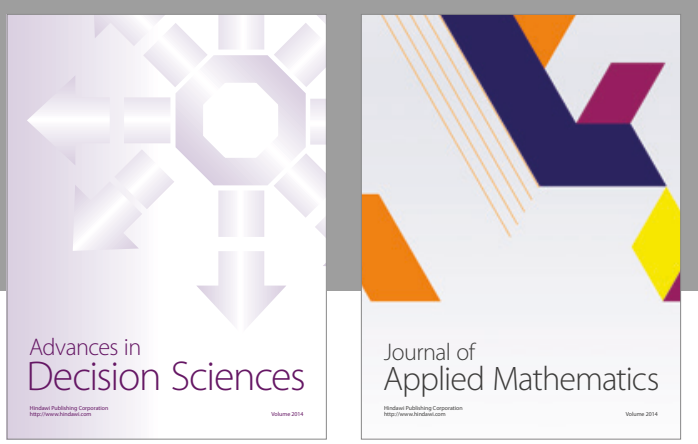

Algebra

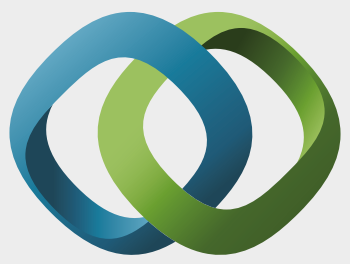

\section{Hindawi}

Submit your manuscripts at

https://www.hindawi.com
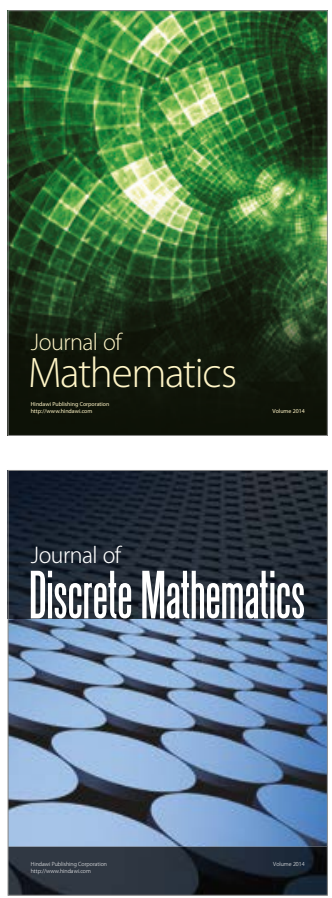

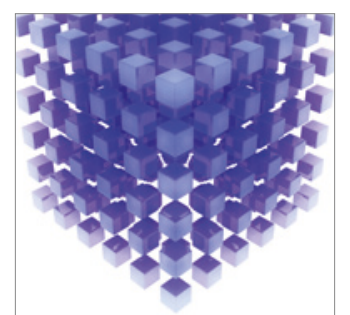

Mathematical Problems in Engineering
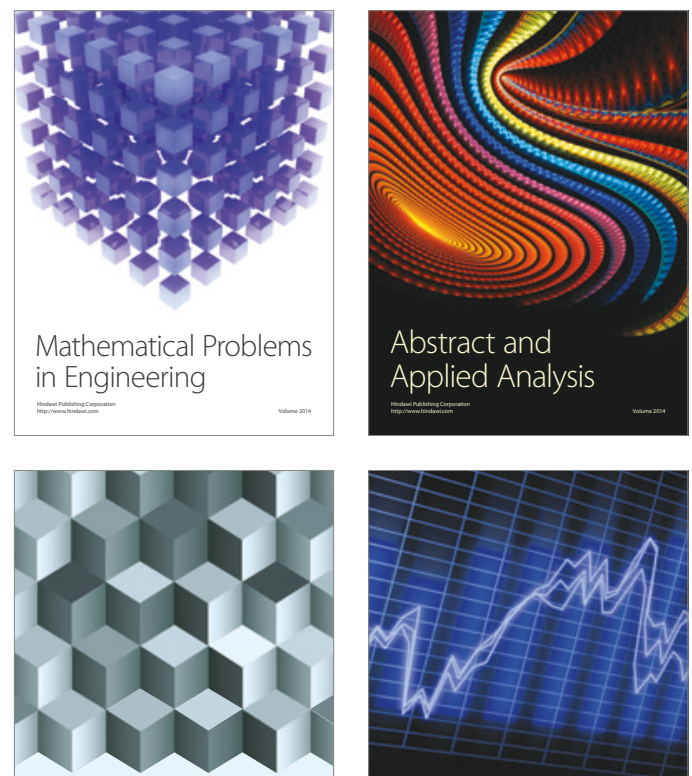

Journal of

Function Spaces

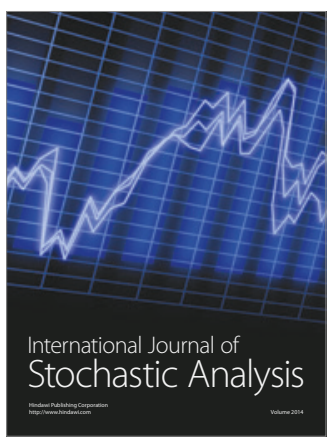

Probability and Statistics
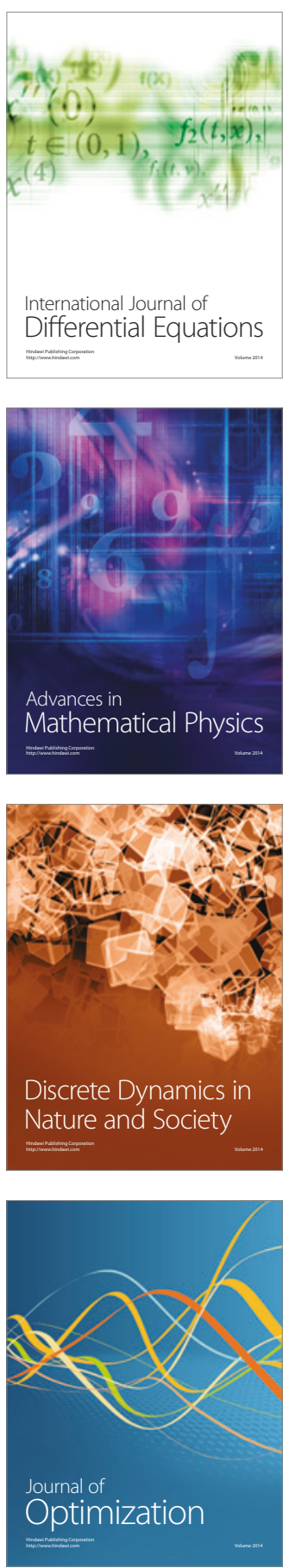